\title{
Can Time-Based Decay Explain Temporal Distinctiveness Effects in Task Switching?
}

\author{
James A. Grange \& Ellen Cross \\ School of Psychology, Keele University
}

In Press: Quarterly Journal of Experimental Psychology

\begin{abstract}
In task switching, extending the response-cue interval (RCI) reduces the switch cost - the detriment to performance when switching compared to repeating tasks. This reduction has been used as evidence for the existence of task-set decay processes. Recently, this has been challenged by the observation of sequential dependencies on the RCI effect: switch cost is only reduced at longer RCIs when the previous trial had a short RCI. This trialwise variation of RCI is thought to affect the temporal distinctiveness (TD) of a previous task's episodic trace, affecting the probability of its automatic retrieval on the current trial; importantly, TD is thought to be independent of the current trial's RCI. The present study highlights a dependency between the current RCI and TD, and demonstrates that a decay model can reproduce some patterns of data attributed to TD. Further, the decay account makes a strong prediction when TD is held constant: repetition response times should slow as RCI increases, and switch response times should be facilitated. This prediction was tested via re-analysis of extant data and three experiments. The re-analysis provided some evidence for the decay account, but Experiments 1 and 2 report slowing for task repetition and switch trials, which cannot be explained by a task-set decay process. Experiment 3, which utilised tasks requiring perceptual judgements, showed small evidence for decay. We conclude that the data are largely consistent with the TD account and that the evidence for decay of higher-level task-sets is not convincing.
\end{abstract}

Keywords: Task switching, decay, temporal distinctiveness

There has been much debate as to whether memory traces decay passively as a function of time, or whether memory traces are forgotten due to proactive interference from competing items (Altmann, 2009; Lewandowsky, Oberauer, \& Brown, 2009a, 2009b; Neath \& Brown, 2012). Evidence from the task switching paradigm (Kiesel et al., 2010; Vandierendonck, Liefooghe, \& Verbruggen, 2010) has claimed to provide several lines of evidence as to the existence of decay processes affecting memory representations of tasks. These so-called task-sets are considered a collection of programmable task parameters (such as attentional 
bias etc.) critical to task performance (Logan \& Gordon, 2001) which must be changed when there is a change in task. According to recent models, successful task performance requires that the relevant task-set is the most active among all competitors (Altmann \& Gray, 2008).

Evidence for decay of task-sets comes from the cuing paradigm, where an explicit cue signals which task is relevant for the current trial. Such paradigms report so-called switch costs - a detriment to response time (RT) and error rates when switching task (i.e., $\mathrm{B} A$ ) compared to repeating the same task (AA). The temporal separation between tasks is manipulated by varying the response-cue interval ( $\mathrm{RCI})$, the time between the response to the previous task and the onset of the cue for the current trial. Several studies have reported a reduction in switch cost at longer RCIs compared to shorter RCIs (see e.g. Meiran, Chorev, \& Sapir, 2000). Decay theories predict such a reduction for two reasons: firstly, on task repetition trials, the previous task's activation will have decayed, reducing its ability to prime performance on the current trial; secondly, on task switch trials, the activation of the previous task (which is irrelevant to the current task) will have decayed, evoking less proactive interference on the current trial (Allport, Styles, \& Hsieh, 1994). The reduction of switch cost with extended RCIs is thus driven by an increase in task repetition RT and a speeding of task switch RT. Decay processes are central to two prominent models of task switching phenomena (Altmann \& Gray, 2008; Schneider \& Logan, 2005); thus, establishing whether task-sets are affected by passive decay is key to assessing the validity of such models.

Evidence against decay of task-sets was presented by Horouchin and colleagues (Horoufchin et al., 2011a; Horoufchin, Philipp, \& Koch, 2011b), who manipulated RCI randomly on a trial-to-trial basis and found that the reduction of switch cost was not a function of absolute time of RCI, but rather was dependent upon the sequential nature of the RCI. Specifically, switch costs were only reduced at long RCIs for the current trialthat is, the RCI between the task at $n-1$ and $n-$ when the RCI for the previous trial (i.e. between tasks at n-2 and n-1) - was short. Additionally, this reduction of switch cost was caused exclusively by a slowing of repetition RTs on these trials; switch RTs were unaffected by RCI manipulations. These findings directly challenge decay theories, which predict a reduction of switch cost at longer RCIs on the current trial independent of the RCI on the previous trial.

Horoufchin et al. (2011a) explained these findings by relating them to a temporal ratio model of serial memory (SIMPLE) proposed by Brown, Neath, and Chater (2007, see Appendix A for further information on this model). When presented with a task cue on the current trial, a retrieval attempt is made of the previous episodic trace of this task; such retrieval has been shown by Brown et al. (2007) to be influenced by the temporal

Please address correspondence to James A. Grange, School of Psychology, Dorothy Hodgkin Building, Keele University, Keele, UK, ST5 5BG. Email: j.a.grange@keele.ac.uk. Part of this work was presented at the Experimental Psychology Society conferences at Lancaster University, UK (April, 2013), and Bangor University, UK (July, 2013). Many thanks are due to Himeh Horoufchin and Iring Koch for providing the raw data from Horoufchin, Philipp, and Koch (2011a) and to George Houghton for fruitful discussion about the modelling presented here. We thank Iring Koch, Andrea Philipp, and five anonymous reviewers for their detailed and constructive comments on previous drafts. 
distinctiveness (TD) of the previous episode. TD refers to the degree to which the previous episode is distinguishable in memory from other episodes presented in close temporal proximity. Traces clustered closely in psychological space will be harder to distinguish, and their subsequent retrieval probability will be low. By varying the RCI from trial-to-trial, Horoufchin et al. (2011a) argued that the TD of task episodes was being affected; operationally, TD was defined as the ratio of the current RCI and the previous trial (i.e. $\mathrm{TD}=$ previous RCI / current RCI).

On task repetition trials, high TD of a task from the previous trial will increase the probability of it being automatically retrieved on the current trial, thus speeding RT due to increased repetition priming. Low $\mathrm{TD}$, on the other hand, reduces the probability of automatic retrieval, and thus task performance does not benefit from repetition priming.

Figure 1 presents the pattern of data that Horoufchin et al. (2011a) presented as evidence of temporal distinctiveness (these data are from the original Horoufchin et al. report). The figure shows decreasing task repetition response time as the RCI-ratio increases, which equates to increased TD. The trend in the data is clear: Repetition RT speeds as the RCI-ratio approaches 1, after which it asymptotes; increasing the RCI-ratio beyond a value of 1 does not appear to produce any additional benefit to RT. This clear dependency of RT on RCI-ratio is strong evidence against passive decay of task-set activation. A formal implementation of this theory - using SIMPLE to estimate retrieval probabilities of tasks - is able to reproduce this data pattern well (see Appendix A and Figure 1).

\section{The Present Study}

Given the importance of the concept of decay to current theories of task switching (e.g., Altmann \& Gray, 2008), it is imperative to explore in detail whether the RCI-ratio effect can in any way be explained by decay processes. Indeed, initially it seems that timebased decay might be a candidate process to explain such patterns of data as in Figure 1, as there appears to be a dependency between RCI ratio and the current trial's absolute value of RCI.

For example, consider Table 1, which highlights the RCIs at $n-1$ and $n-2$ that make up each RCI-ratio for the two groups of Experiment 1 in Horoufchin et al. (2011a). Although the RCI-ratios are thought to be independent of the current trial's RCI, higher RCI-ratios tend to have shorter RCIs on the current trial, compared to lower RCI-ratios which tend to have longer RCIs on the current trial. As a decay process predicts faster RTs with decreasing RCI for task repetitions (due to increased repetition priming; Altmann \& Gray, 2008) the facilitation to RT at increasing RCI-ratios might be caused by these trials having shorter - on average - RCIs on the current trial. Thus, the pattern attributed to temporal distinctiveness might be able to be explained by a pure decay-based process. Indeed, as can be seen in Figure 1, a decay model can reproduce this pattern of data (see Appendix B for details of the model).

It is clear from Figure 1 that there is some degree of mimicry between the competing accounts of the RCI-ratio data, in that they both predict facilitated RT at extended RCIratios, explained using opposing theoretical constructs. However, it turns out that the accounts make contrary predictions when the RCI-ratios are at unity (i.e., an RCI-ratio of 1). Specifically, in Table 1 there are four exemplars of RCIs at $n-1$ and $n-2$ that produce an RCI-ratio of 1: (e.g. for Group 1) 100-100; 200-200; 1,000-1,000; and 2,000-2,000. As 


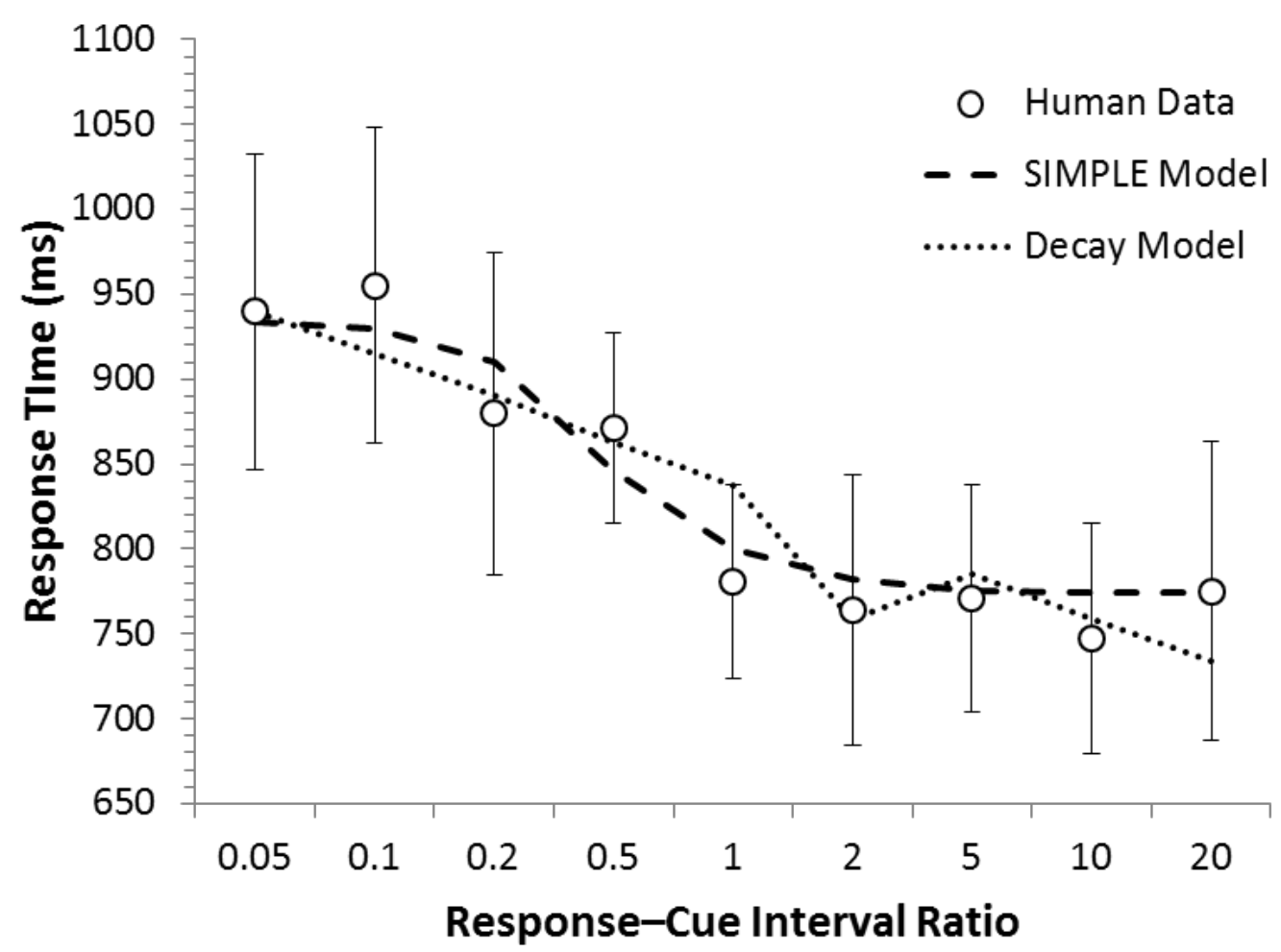

Figure 1. Human response times (in milliseconds, ms) from Horoufchin et al. (2011a) (Experiment 1, Group 1) and model predictions as a function of RCI-ratio for the SIMPLE (i.e. temporal distinctiveness) model and decay model. Error bars represent $95 \%$ confidence intervals around the human data mean.

the ratio is 1 , the temporal distinctiveness account of Horoufchin et al. (2011a) predicts identical performance regardless of the current trial's RCI (see Appendix A), as the tobe-retrieved memory has the same temporal distinctiveness in each case; however, a decay account would predict slowing of repetition response times at longer RCIs on the current trial and speeding of switch RT. Thus this scenario allows us to explore the effects of timebased decay (if any) when interference is held constant, and therefore provides opportunity to falsify the decay account ${ }^{1}$.

The purpose of the present study is to investigate this critical prediction in an attempt to differentiate between the temporal distinctiveness account and the decay account. We test this prediction in re-analysis of Horoufchin et al. (2011a) and three experiments: Experiment 1 was a close replication of Horoufchin et al. and Experiment 2 was a conceptual replication; Experiment 3 was a close replication of Meiran et al. (2000). Although we find some evidence supporting decay in the re-analysis, we find no evidence in support of decay in Experiments 1 or 2; instead, the data appears consistent with TD. In Experiment 3-which

\footnotetext{
${ }^{1}$ Note that there are other scenarios in Table 1 where the current trial's RCI differs but the overall TD is constant. For example, 100-1000 and 200-2000 both have an RCI-ratio of 0.1, but the current RCIs are $1000 \mathrm{~ms}$ and $2000 \mathrm{~ms}$, respectively. Thus, this scenario also allows one to examine decay whilst controlling for TD. However, the number of examplars of fixed RCI-ratio and varying RCI is greatest for RCI-ratio of 1 (i.e., unity), and therefore it is exclusively this scenario which is examined in this paper.
} 
Table 1

RCI-ratios for both groups from Horoufchin et al. (2011a), together with the sequential RCIs at n-1 and n-2 that make up each ratio.

\begin{tabular}{cccccc}
\hline \multicolumn{3}{c}{ Group 1 } & \multicolumn{4}{c}{ Group 2 } \\
\hline RCI-Ratio & $\mathrm{n}-1$ & $\mathrm{n}-2$ & RCI-Ratio & $\mathrm{n}-1$ & $\mathrm{n}-2$ \\
\hline 0.05 & 2000 & 100 & 0.05 & 1000 & 50 \\
0.1 & 2000 & 200 & 0.15 & 1000 & 150 \\
& 1000 & 100 & 0.17 & 300 & 50 \\
0.2 & 1000 & 200 & 0.3 & 1000 & 300 \\
0.5 & 200 & 100 & 0.33 & 150 & 50 \\
& 2000 & 1000 & 0.5 & 300 & 150 \\
1 & 100 & 100 & 1 & 50 & 50 \\
& 200 & 200 & & 150 & 150 \\
& 1000 & 1000 & & 300 & 300 \\
& 2000 & 2000 & & 1000 & 1000 \\
2 & 100 & 200 & 2 & 150 & 300 \\
& 1000 & 2000 & 3 & 50 & 150 \\
5 & 200 & 1000 & 3.33 & 300 & 1000 \\
10 & 100 & 1000 & 6 & 50 & 300 \\
& 200 & 2000 & 6.67 & 150 & 1000 \\
20 & 100 & 2000 & 20 & 50 & 1000 \\
\hline
\end{tabular}

utilises tasks emphasising perceptual judgements - we find some evidence for decay. On the basis of these findings, we form a conjecture that higher-level task sets are not subject to decay, but perceptual representations might be (see also Horoufchin et al., Experiment 4).

\section{Horoufchin et al. (2011a) re-analysis}

In this section, we re-analyse the data reported by Horoufchin et al. (2011a) by examining the effect of the current trial's RCI on response time when the RCI-ratio is at unity. The general analytical strategy employed in this paper follows that recommended by Cumming (2014) of using estimation of effect sizes (and their associated confidence intervals, CIs) rather than null hypothesis significance testing.

For the purposes of the present study, we report point estimates of response times (and error rates for the three reported experiments), together with 95\% CIs, which provides a measure of the precision of these point estimates. Then, in order to examine the predictions of the decay account, the effect size of interest becomes difference scores in RT between successive RCIs; in such cases, we report these effect sizes as well as the CI of these differences (Cumming, 2014). To aid the reader less familiar with this approach, we provide comprehensive discussion regarding interpretation of this type of analysis for the data reported in this first section.

The data from Horoufchin et al. (2011a, Experiment 1, Group 1) when the RCI-ratio is at unity is shown in Figure 2. The mean RTs (and CIs) for repetition and switch trials across all RCIs are shown in Figure $2 \mathbf{A}$. This figure shows a large switch cost which seems 
to reduce at extended RCIs; this appears to be due to a slowing of task repetition RT with increasing RCI, and a speeding of switch RT, the pattern predicted by a decay account. These observations are confirmed by the other elements in the Figure. Figure 2 B plots the switch cost (switch RT - repetition RT) for each RCI. The switch cost is very large at the shortest RCI (364ms, 95\% CI [244, 484]) which reliably reduced to a (still sizable) switch cost of $154 \mathrm{~ms}[86,222]$ at the longest RCI. To examine the tests of the decay account, we now consider task repetition and task switch trials separately. We examine the change in RT between successive pairs of RCIs (i.e., 100-200ms, 200-1000ms, and 1000-2000ms). We thus calculate difference scores for each sequence and each RCI, as well as the $95 \%$ CIs of these differences (Cumming, 2014). A decay model would predict positive differences in repetition RT at each RCI-pair, as the task-set should be decaying and therefore less able to prime performance. Switch RT should show negative differences at each RCI-pair, as the interference from the previous task should reduce with increasing RCI due to its representation decaying. Difference scores in the Figure whose CIs include zero do not provide convincing evidence for differences in means.

Figure $2 \mathbf{C}$ shows task repetition trials; there was very little evidence of slowing between RCIs of $100 \mathrm{~ms}-200 \mathrm{~ms}$ or between $200 \mathrm{~ms}-1000 \mathrm{~ms}$; there was however modest slowing of $72 \mathrm{~ms}[6,138]$ between RCIs of $1000 \mathrm{~ms}-2000 \mathrm{~ms}$. For task switch trials (Figure $2 \mathrm{D})$ the difference in RT between each successive RCI was very small, with zero being a credible estimate for each difference.

This re-analysis has highlighted potential evidence of a decay process, although the effect sizes are very small. There were very small measures of slowing for task repetition trials as RCI increased, and negligible speeding for task switch trials. However, the overall pattern of data is compatible with a decay account.

\section{Experiment 1}

To further investigate the effect of the current trial's RCI when the RCI-ratio is at unity, we conducted a close replication of Experiment 1 (Group 1) of Horoufchin et al. (2011a). The purpose of this experiment is to examine whether the effect reported in the post-hoc analysis above replicates.

\section{Method}

Participants. 36 participants were recruited from the participant panel run by the School of Psychology at Keele University in exchange for partial course credit. 5 participants were removed for session-wise accuracy below $90 \%$.

Apparatus \& Stimuli. Stimuli were presented on a 17 -inch monitor, connected to a PC running E-Prime v. 2.0 software. Responses were collected with a 1-ms precise USB keyboard. The task involved switching between two possible judgements on bivalent stimuli. The stimuli consisted of the letter $\mathrm{A}$ and the number 4, which was presented either in red or blue font. The two possible tasks were a form judgement (i.e. judge whether the stimulus is an A vs. 4) and a colour judgement (i.e. judge whether the stimulus is red vs. blue). The stimuli were presented on a grey background, within a rectangular frame with a width of $4 \mathrm{~cm}$ and a height of $3.5 \mathrm{~cm}$. The relevant task on each trial was cued by 4 shapes presented on the outside corner of the rectangular frame: black dollar signs cued the form 

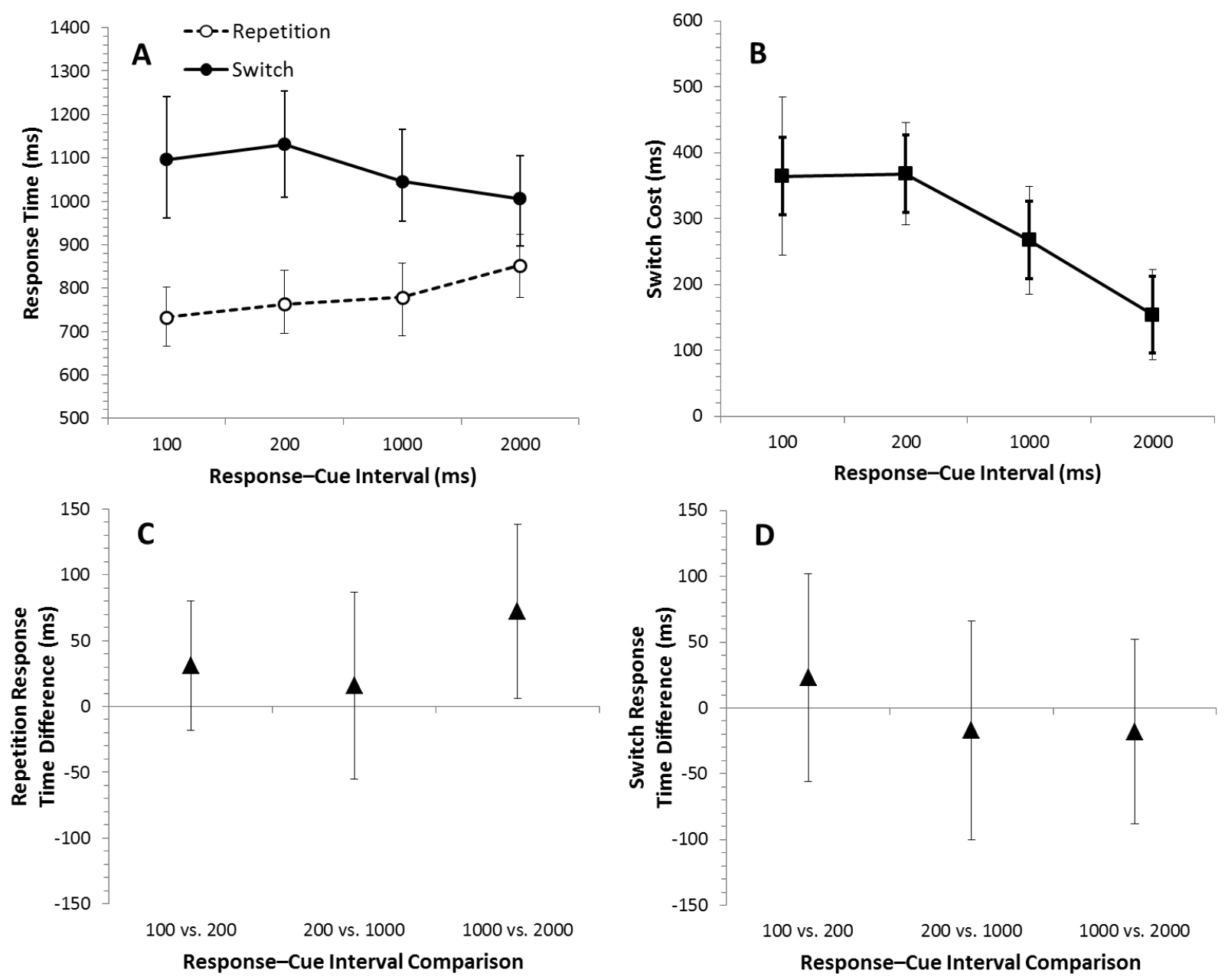

Figure 2. Re-analysis of data from Horoufchin et al. (2011) where the RCI-ratio is at unity. A. Mean response times (in milliseconds, ms) for task repetition and task switch trials as a function of response-cue interval. Error bars denote $95 \%$ confidence intervals. B. Mean switch costs as a function of response-cue interval. Bold error bars represent +/95\% within-subject confidence intervals (Loftus \& Masson, 1994); thin error bars represent standard 95\% confidence intervals. C. Pairwise comparisons showing mean differences as response-interval increases for task repetition trials, and D. shows switch trials. Error bars denote $95 \%$ confidence intervals. 
task, and yellow squares cued the colour task. The cues were $0.7 \mathrm{~cm}$ in width and height. Response keys were the $\mathrm{Z}$ and the $\mathrm{M}$ keys on the keyboard; Z corresponded to "letter" and "red" responses, and M corresponded to "number" and "blue" responses. Similar to Horoufchin et al. (2011a), reminders of the response mappings were made available on an instruction sheet to participants throughout the whole experiment.

Procedure. The experiment presented 8 blocks of 96 experimental trials, preceded by 20 practice trials. Each trial consisted of the presentation of the relevant cue for the current task; cues were presented for $100 \mathrm{~ms}$, before the target appeared centered within the rectangular frame. The cues remained visible together with the target until a response was registered by the participant. Once a response had been executed, the target and cues disappeared for a random RCI taken from the set of possible RCIs: 100ms, 200ms, $1,000 \mathrm{~ms}$ or $2,000 \mathrm{~ms}$. The relevant task and RCI for the next trial was selected randomly (without replacement) from the total set of permuted tasks, stimuli, and RCI values. No error feedback was provided (cf., Horoufchin et al., 2011a).

Design. The experiment manipulated two factors in each group in a fully related design: Task sequence (switch vs. repetition) and $R C I$ (100ms, 200ms, 1,000ms, and 2,000ms). The dependent variable throughout was response time (RT) in milliseconds (ms) and percentage error $(\%)$.

\section{Results}

The first two trials from each block were not analysed, and for the RT analysis neither were error trials or the two trials following an error. Furthermore, RTs greater than 2.5 standard deviations from each participants' mean for each cell of the experimental design were considered outliers and were removed.

Although participants made fewer errors on task repetition trials than task switch trials $(-0.99 \%[-2.00,0.03])$, there were no clear effects of RCI or an interaction in the error rates (see Table 2 ).

Table 2

Mean error rates (\%) for Experiment 1 with 95\% confidence intervals in square brackets, for both the global analysis and the RCI-ratio unity analysis.

\begin{tabular}{ccccc}
\hline & \multicolumn{2}{c}{ Global Analysis } & \multicolumn{2}{c}{ RCI-Ratio Unity } \\
\hline RCI $(\mathrm{ms})$ & Repetition & Switch & Repetition & Switch \\
\hline 100 & $2.99[2.10,3.87]$ & $4.09[2.65,5.54]$ & $3.92[1.81,6.04]$ & $4.31[2.68,5.95]$ \\
200 & $3.45[2.61,4.29]$ & $4.39[3.08,5.69]$ & $3.43[2.16,4.70]$ & $3.10[1.55,4.65]$ \\
1,000 & $3.52[2.41,4.63]$ & $3.90[2.48,5.69]$ & $3.02[1.10,4.94]$ & $3.54[1.81,5.27]$ \\
2,000 & $2.96[1.81,4.12]$ & $4.48[3.03,5.93]$ & $3.35[1.50,5.21]$ & $4.70[3.27,6.07]$ \\
\hline
\end{tabular}

The mean RTs for Experiment 1 can be seen in Figure $3 \mathbf{A}$. The figure shows that as RCI increases, RT for task switch and task repetition trials increase. There is a clear cost to switching tasks for all RCIs; the magnitude of these switch costs are shown in Figure 3 B, which show positive and large switch costs for all RCIs. This figure also shows no strong reduction of switch cost with extended RCI, contrary to the pattern expected by a decay account (Meiran et al., 2000) and the account of Horoufchin et al. (2011a); there was no 
change in the switch cost between RCIs of 100,200 , and $1000 \mathrm{~ms}$, but a slight reduction in the cost at the RCI of $2000 \mathrm{~ms}$.

Although a decay account predicts a reduction due to decreasing switch RTs and increasing repetition RTs, Figure $3 \mathbf{A}$ shows that RTs tend to slow at extended RCIs for repetition and switch trials. Figures $3 \mathbf{C}$ and $\mathbf{D}$ show that - for repetition and switch trials respectively - there is little slowing between the RCIs of $100 \mathrm{~ms}$ and $200 \mathrm{~ms}$, but large slowing between $200 \mathrm{~ms}-1000 \mathrm{~ms}$ and between $1,000 \mathrm{~ms}-200 \mathrm{~ms}$.

The RCI-ratio data can be seen in Figure 4. Similar to Horoufchin et al. (2011a), RTs became faster as the RCI-ratio increased (Figure 4 A). However, this facilitation to RT was not limited to repetition trials; the Figure clearly shows that switch trials too benefited from a larger RCI-ratio, although the facilitation does seem to be greater for repetition trials. Figure $4 \mathbf{B}$ shows that the difference in RT between switch and repetition RTs across the RCI ratios was generally positive and large, with the exception of the 0.05 ratio; the rate of facilitation was greater for repetition trials between the ratios of 0.05 and 0.2 , after which the difference was relatively stable.

Ratio-unity analysis. The ratio-unity analysis only considered those trials where the RCI-ratio was at unity. Figure 5 shows the RTs for this subset of data, and Table 2 shows the error rates. For the errors, there were no clear effects between any of the conditions of interest, so the analysis focusses on the RTs.

The pattern of RT data mirrored that of the main analysis, with large switch costs for all RCI comparisons (Figure 5 B), with the exception of the final RCI, where zero switch cost is a credible estimate. Figure $5 \mathbf{A}$ again shows slowing at extended RCIs. Figures 5 $\mathbf{C}$ and $\mathbf{D}$ suggests this slowing is similar for repetition and switch trials, showing that - for repetition and switch trials - there is little slowing between the RCIs of $100 \mathrm{~ms}$ and $200 \mathrm{~ms}$, but there is modest slowing between $200 \mathrm{~ms}-1000 \mathrm{~ms}$ and between $1000 \mathrm{~ms}-2000 \mathrm{~ms}$.

\section{Discussion}

The present experiment successfully replicated some key effects from Horoufchin et al. (2011a), although there were also some differences. First, the present experiment did not observe a large decrease of switch cost as the RCI increased. This reduction has been reported by several authors (Horoufchin et al., 2011a; Meiran et al., 2000), although some authors have not reported such a decrease under some conditions (Altmann, 2005). Such a reduction is typically caused by the net effect of decreasing switch response time and increasing repetition response time with increasing RCI. However, the present study observed slowing for both repetition and switch response time as RCI increased, which is an atypical finding in the task switching literature. We return to the discussion of this discrepancy in the General Discussion.

The present study did observe a large decrease in response time as the RCI-ratio increased, which is consistent with the temporal distinctiveness account of Horoufchin et al. (2011a). However, the present study also observed facilitation to RT for task switch trials. As Figure $4 \mathbf{B}$ shows, the rate of facilitation was larger for repetition trials between ratios of 0.05 and 0.2 , but similar for repetition and switch trials between ratios of 0.2 and 20, suggesting that - in the present study at least - ratio effects are not limited to task repetition trials. 

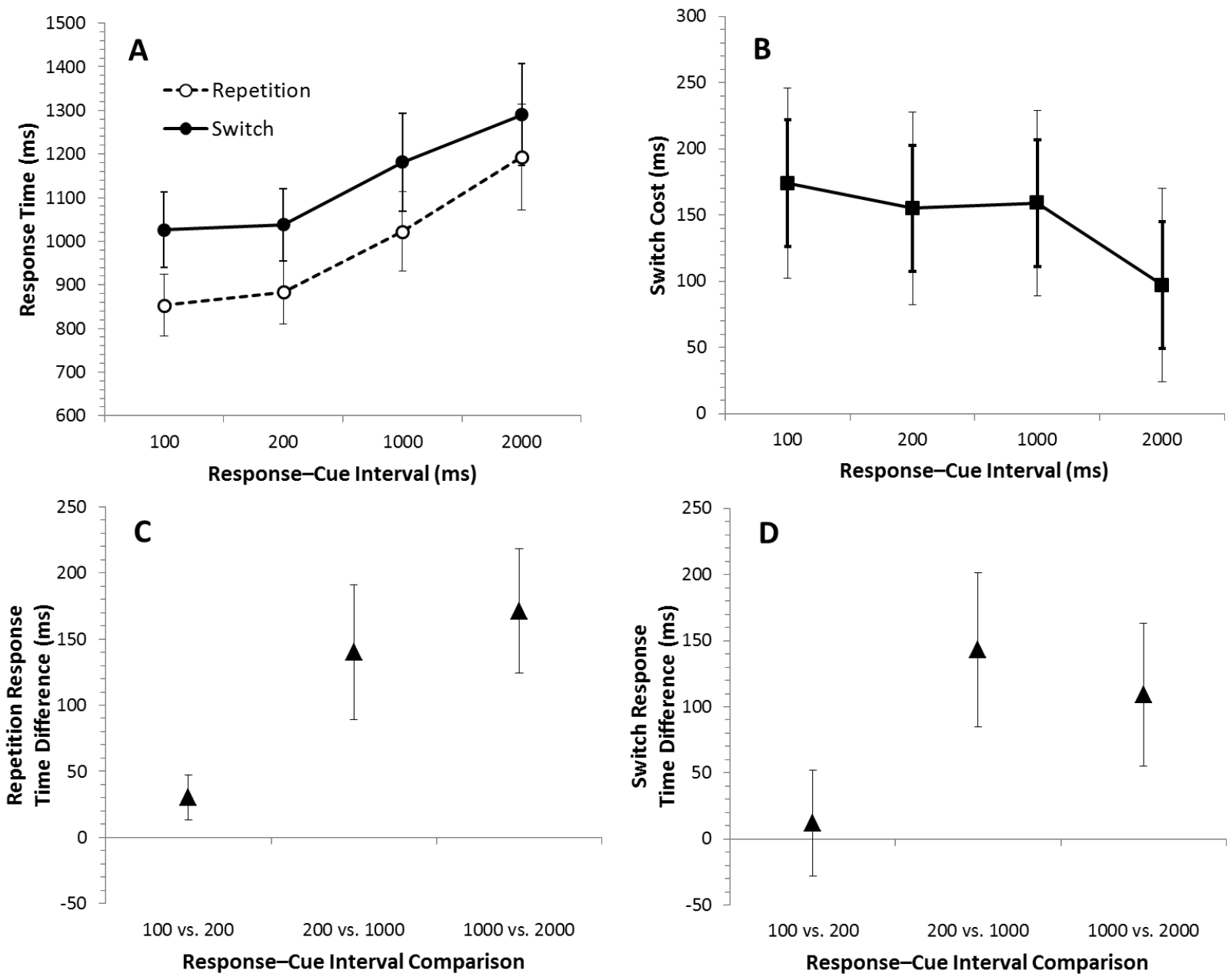

Figure 3. Data from Experiment 1. A. Mean response times (in milliseconds, ms) for task repetition and task switch trials as a function of response-cue interval. Error bars denote $95 \%$ confidence intervals. B. Mean switch costs as a function of response-cue interval. Bold error bars represent $+/-95 \%$ within-subject confidence intervals using the error term from the Sequence * RCI interaction (Loftus \& Masson, 1994); thin error bars represent standard $95 \%$ confidence intervals. C. Pairwise comparisons showing mean differences as response cue interval increases for task repetition trials, and D. for switch trials. Error bars denote $95 \%$ confidence intervals. 

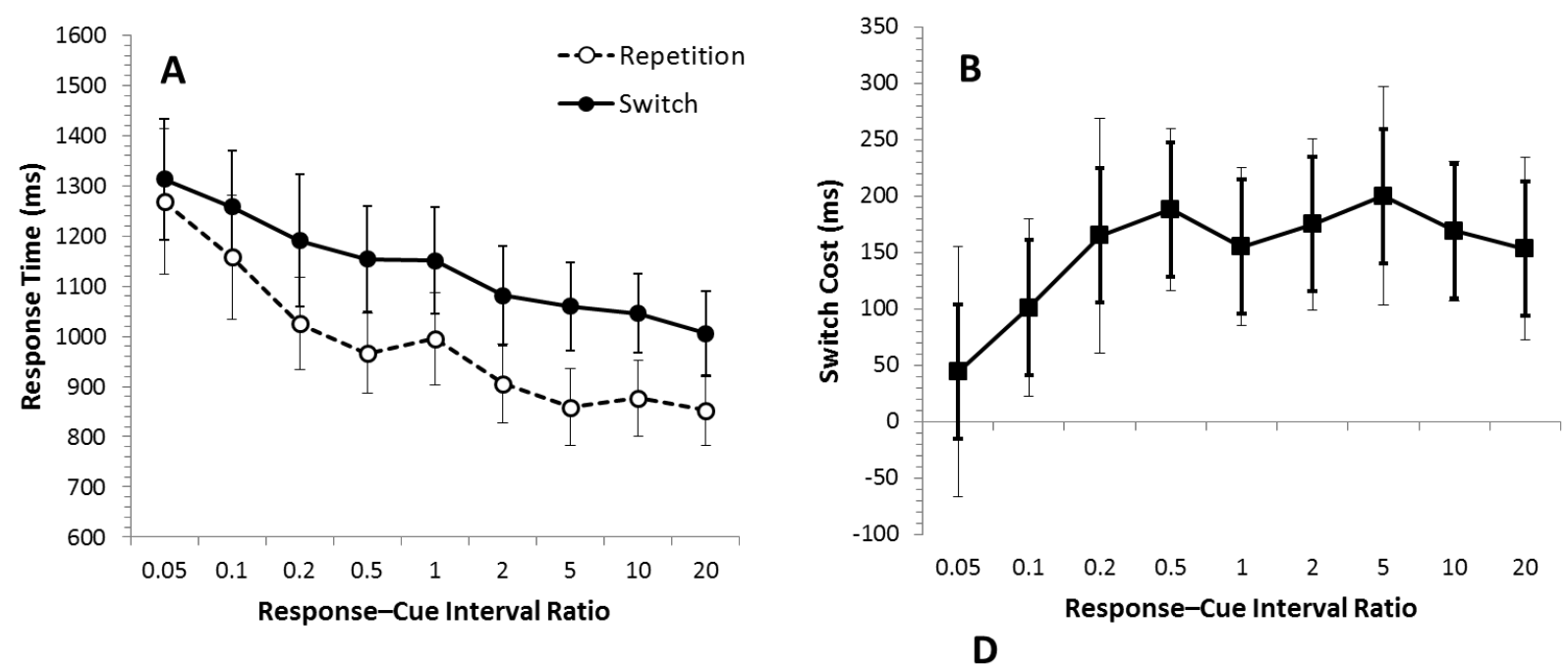

Figure 4. Data from Experiment 1. A. Mean response times (in milliseconds, ms) for task repetition and task switch trials as a function of response-cue interval ratio. Error bars denote $95 \%$ confidence intervals. B. Mean switch costs as a function of response-cue interval ratio. Bold error bars represent $+/-95 \%$ within-subject confidence intervals using the error term from the Sequence * RCI-ratio interaction (Loftus \& Masson, 1994); thin error bars represent standard $95 \%$ confidence intervals.

Critically, the decay account was not supported by the RCI-ratio unity analysis, which demonstrated slowing for repetition and switch trials as the current trial's RCI increased. This result cannot be explained by a pure decay account of task-sets, which would only predict slowing for repetition trials (switch trials should become facilitated); that slowing is observed on switch trials too is strong evidence against the decay account.

\section{Experiment 2}

The observed slowing of task switch response time with increasing RCI is not only not predicted by a decay account, it is also quite an atypical finding in the task switching literature. Although studies investigating the effects of RCI on task switching performance report increasing repetition RTs (but see Altmann, 2005), switch trials are generally reported to decrease (Horoufchin et al., 2011a, 2011b); however, in the study of Meiran et al. (2000) the first study to report a reduction of switch cost with extended RCI - switch trials were generally unaffected by increasing RCI. Thus, the observed increase of task switch RT with increasing RCI in the present experiment is certainly atypical and in need of replication.

Thus, for Experiment 2 we again manipulated RCI in a task switching paradigm, but the task demands were altered from that of Experiment 1. In Experiment 2, participants were presented with a centrally-positioned digit, the colour of which determined which task to perform. In addition, we reduced the number of RCI values to three, and increased the total number of trials; this increases the number of trials per cell of the experimental design, which becomes important when analysing RTs for RCI-ratio unity trials, which only analyses an infrequent subset of the data. Increasing the number of trials for this analysis 

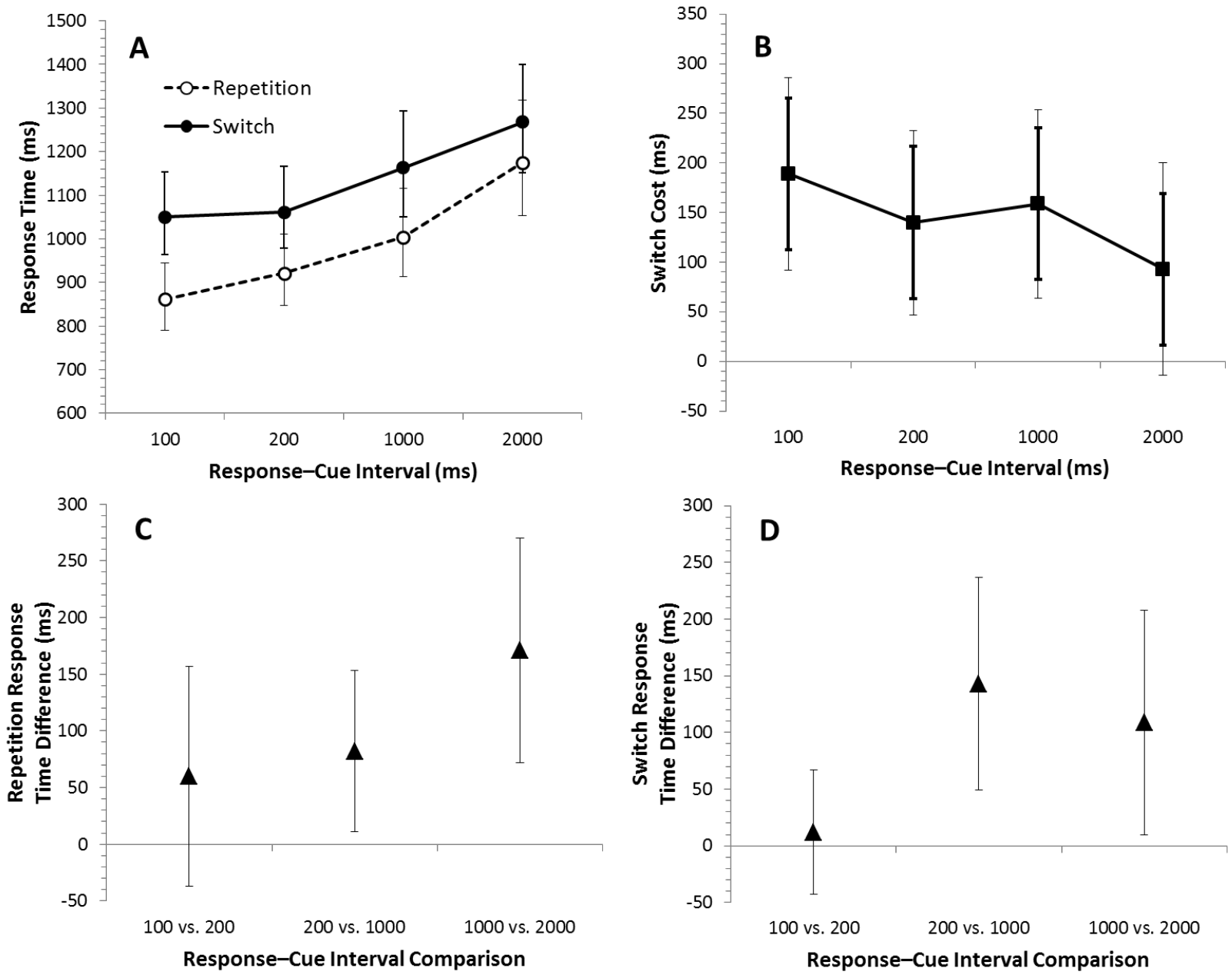

Figure 5. Data from Experiment 1 when the response-cue interval ratio is at unity. A. Mean response times (in milliseconds, ms) for task repetition and task switch trials as a function of response-cue interval. Error bars denote $95 \%$ confidence intervals. B. Mean switch costs as a function of response-cue interval. Bold error bars represent $+/-95 \%$ within-subject confidence intervals using the error term from the Sequence * RCI interaction (Loftus \& Masson, 1994); thin error bars represent standard 95\% confidence intervals. C. Pairwise comparisons showing mean differences as response-cue interval increases for task repetition trials, and D. for switch trials. Error bars denote 95\% confidence intervals. 
increases the precision of the point estimates (cf., the relatively wide CIs in Figures $5 \mathbf{C}$ and $\mathbf{D})$.

\section{Method}

Participants. 40 new participants were recruited from the same pool as Experiment 1; 9 were excluded due to session-wise accuracy below $90 \%$.

Stimuli \& Procedure. The same experimental apparatus was used as in Experiment 1 . On each trial, participants were presented with centrally-positioned fixation cross presented in size 16 black Times-New Roman font on a grey background. After a variable response-cue interval, the fixation cross turned either red or blue (with equal probability), and remained visible for a further $100 \mathrm{~ms}$, after which time a digit (randomly selected from the set 1-9, excluding 5) of the same colour replaced the cross. If the colour was blue, participants were required to make an odd/even judgement, and if it was red, participants were required to make a lower/higher than 5 judgement. The response key "Z" was used for a lower or odd response, and "M" was used for a higher or even response. The experiment consisted of 12 blocks of 96 trials, preceded by a practice block of 20 trials.

Only three RCIs were used in the current study. To ensure accuracy of stimulus presentation times, RCIs were set so as to coincide with screen refresh rates on the experimental monitors $(85.02 \mathrm{~Hz})$. Thus, RCIs of $84 \mathrm{~ms}, 390 \mathrm{~ms}$, and $990 \mathrm{~ms}$ were used.

Design. The experiment manipulated two factors in each group in a fully related design: Task sequence (switch vs. repetition) and $R C I(84 \mathrm{~ms}, 390 \mathrm{~ms}$, and $990 \mathrm{~ms})$. The dependent variables throughout were again $\mathrm{RT}$ and error rates.

\section{Results}

Data trimming was identical to that of Experiment 1. Mean error rates are shown in Table 3, which showed fewer errors on task repetition trials than task switch trials $(-1.62 \%$ $[-2.20,-1.06]$. This difference appeared to dissipate at extended RCIs: $-2.49 \%$ [-3.36, 1.62], $-2.06 \%$ [-2.98, -1.14], $-0.34 \%$ [-1.18, 0.51] for RCIs 84, 390, and 990ms respectively. This was caused by a very small increase in task repetition error rates between RCIs of $84-390 \mathrm{~ms}$ $(0.36 \%[-0.23,0.95])$ and between RCIs of $390-990 \mathrm{~ms}(0.54 \%[-0.19,1.26])$; for switch trials, there was no change in error rates between RCIs of $84-390 \mathrm{~ms}(-0.07 \%[-0.95,0.81])$ but a large decrease of errors between RCIs of 390-990ms (-1.19\% [-1.98, -0.39]).

Table 3

Mean error rates (\%) for Experiment 2 with 95\% confidence intervals in square brackets, for both the global analysis and the RCI-ratio unity analysis.

\begin{tabular}{ccccc}
\hline & \multicolumn{2}{c}{ Global Analysis } & \multicolumn{2}{c}{ RCI-Ratio Unity } \\
\hline RCI $(\mathrm{ms})$ & Repetition & Switch & Repetition & Switch \\
\hline 84 & $2.83[2.20,3.45]$ & $5.32[4.33,6.30]$ & $3.04[2.14,3.94]$ & $5.47[4.04,6.89]$ \\
390 & $3.19[2.53,3.85]$ & $5.25[4.19,6.31]$ & $3.79[2.57,5.01]$ & $5.46[3.87,7.06]$ \\
990 & $3.73[2.77,4.68]$ & $4.06[3.25,4.87]$ & $4.36[3.12,5.60]$ & $4.44[3.35,5.52]$ \\
\hline
\end{tabular}

The RTs for Experiment 2 are shown in Figure 6. As can be seen in Figure 6 A, there was a large switch cost and general slowing of RTs as RCI increased. As can be seen 
in Figure $6 \mathbf{B}$, no clear reduction of switch cost with increasing RCI was observed. For repetition trials, Figure $6 \mathbf{C}$ shows small slowing between the RCIs of $84 \mathrm{~ms}$ and $390 \mathrm{~ms}$, but large slowing between RCIs of 390ms and 990s; Figure 6 D shows that this general pattern was also found for task switch trials (although with a smaller amount of slowing between 84-390ms), replicating the finding of equivalent slowing of RTs with increasing RCI for both repetition and switch trials found in Experiment 1.

The RTs as a function of RCI-ratio can be seen in Figure 7. Figure $7 \mathbf{A}$ shows again a large switch cost and general facilitation to performance with increasing RCI-ratio. Figure $7 \mathbf{B}$ shows no clear increase of switch cost with increasing RCI-ratio.

Ratio-unity analysis. Mean RCI-ratio unity error rates are shown in Table 3, which showed fewer errors on task repetition trials than task switch trials $(-1.39 \%[-2.29$, -0.50]. This difference appeared to decrease at extended RCIs: $-2.42 \%[-3.88,-0.97],-1.67 \%$ $[-3.43,0.08]$, and $-0.08 \%[-1.49,1.33]$ for RCIs 84,390 , and $990 \mathrm{~ms}$ respectively. This was caused by a very small increase in task repetition error rates between RCIs of $84-390 \mathrm{~ms}$ $(0.75 \%[-0.46,1.96])$ and between RCIs of $390-990 \mathrm{~ms}(0.57 \%[-0.80,1.94])$; for switch trials, there was no change in error rates between RCIs of $84-390 \mathrm{~ms}(-0.002 \%[-1.85,1.86])$ but a small decrease of errors between RCIs of 390-990ms (-1.02\% [-2.89, 0.83]).

The RT data when the RCI-ratio is at unity is shown in Figure 8. Aside from the large switch cost, there was again slowing as the RCI increased, with a reduction of the switch cost only for the intermediate RCI (see Figure $8 \mathbf{B}$ ). Figures $8 \mathbf{C}$ and $\mathbf{D}$ show that this occurred due to relatively large slowing for task repetition trials between RCIs of $84 \mathrm{~ms}$ and $390 \mathrm{~ms}$, but no slowing for task switch trials. However, there was large slowing between RCIs of $390 \mathrm{~ms}$ and $990 \mathrm{~ms}$ for both repetition and switch trials, in contrast to the decay predictions.

\section{Discussion}

The results from Experiment 2 largely replicated those of Experiment 1, in that for the RCI-ratio unity analysis, RTs slowed for repetition and switch trials, which is not predicted by a decay account. There was a decay-like pattern in the error data, in that switch cost reduced at extended RCIs due to very small increase of task repetition errors and moderate decrease of task switch errors. However, that this pattern in the switch error rates was opposite that of the switch-RT data suggests this was due to a speed-accuracy trade-off (the cause of which is not clear). This pattern of data does not provide convincing evidence for decay of task sets.

Thus far, the evidence for decay in explaining the RCI effect in task switching is not convincing. A decay account predicts slowing only for task repetition RT as RCI increases (switch RT should become facilitated); both Experiments 1 and 2 find evidence of slowing for task repetition and task switch trials, which cannot be explained by decaying task representations.

However, Horoufchin et al. (2011a) presented some evidence for decay independent of temporal distinctiveness. Using two cues per task, Horoufchin et al. (2011a, Experiment 4) showed that the cue-switch cost - the RT cost of switching cues without the task itself switching (Logan \& Bundesen, 2003; Mayr \& Kliegl, 2003) - decreased at extended RCIs independent of RCI-ratio; this suggests that some representation of the cue itself decays as a function of time, and is not influenced by TD. Indeed, one model of task switching 

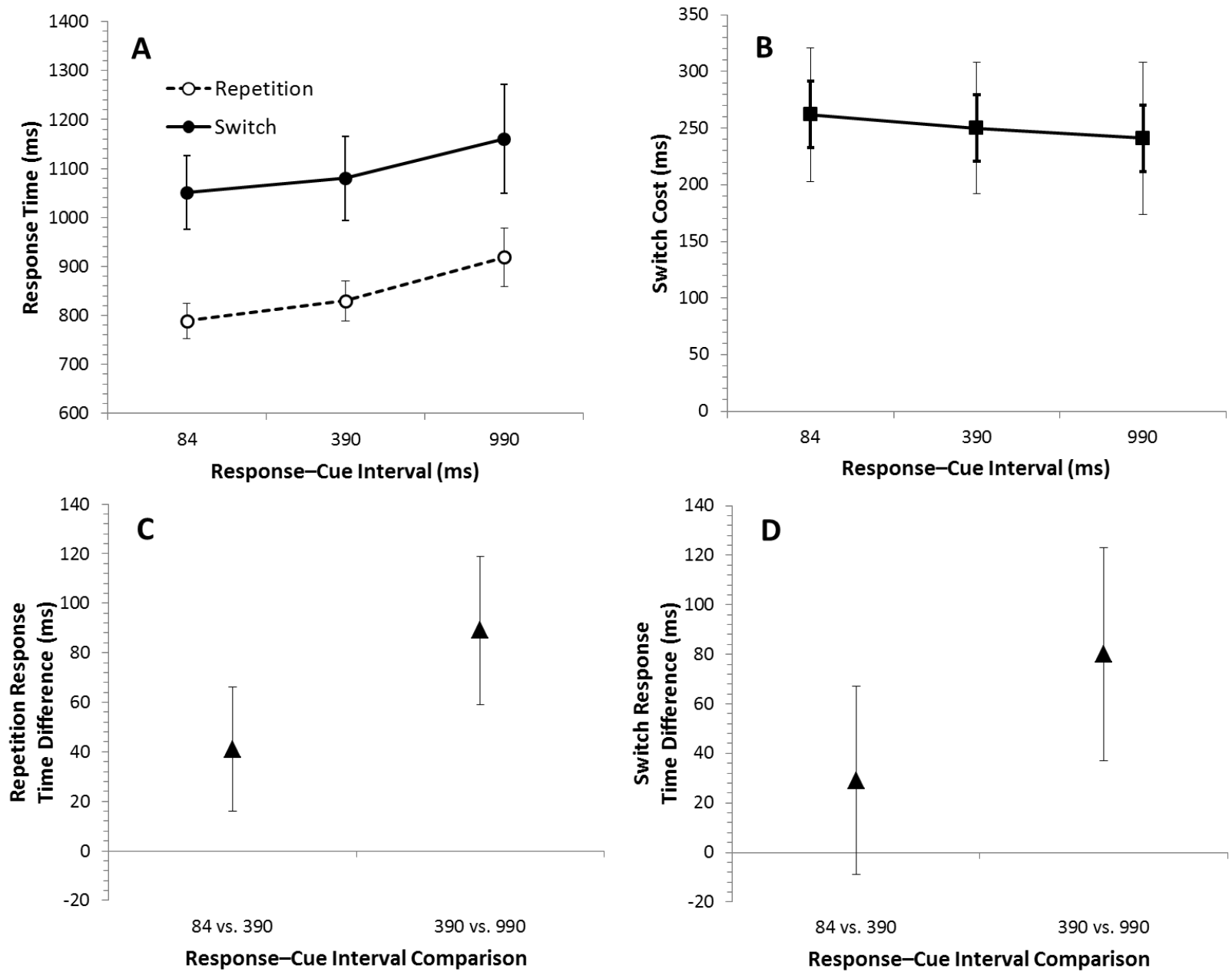

Figure 6. Data from Experiment 2. A. Mean response times (in milliseconds, ms) for task repetition and task switch trials as a function of response-cue interval. Error bars denote $95 \%$ confidence intervals. B. Mean switch costs as a function of response-cue interval. Bold error bars represent $+/-95 \%$ within-subject confidence intervals using the error term from the Sequence * RCI interaction (Loftus \& Masson, 1994); thin error bars represent standard 95\% confidence intervals. C. Pairwise comparisons showing mean differences as responsecue interval increases for task repetition trials, and D. for switch trials. Error bars denote $95 \%$ confidence intervals. 

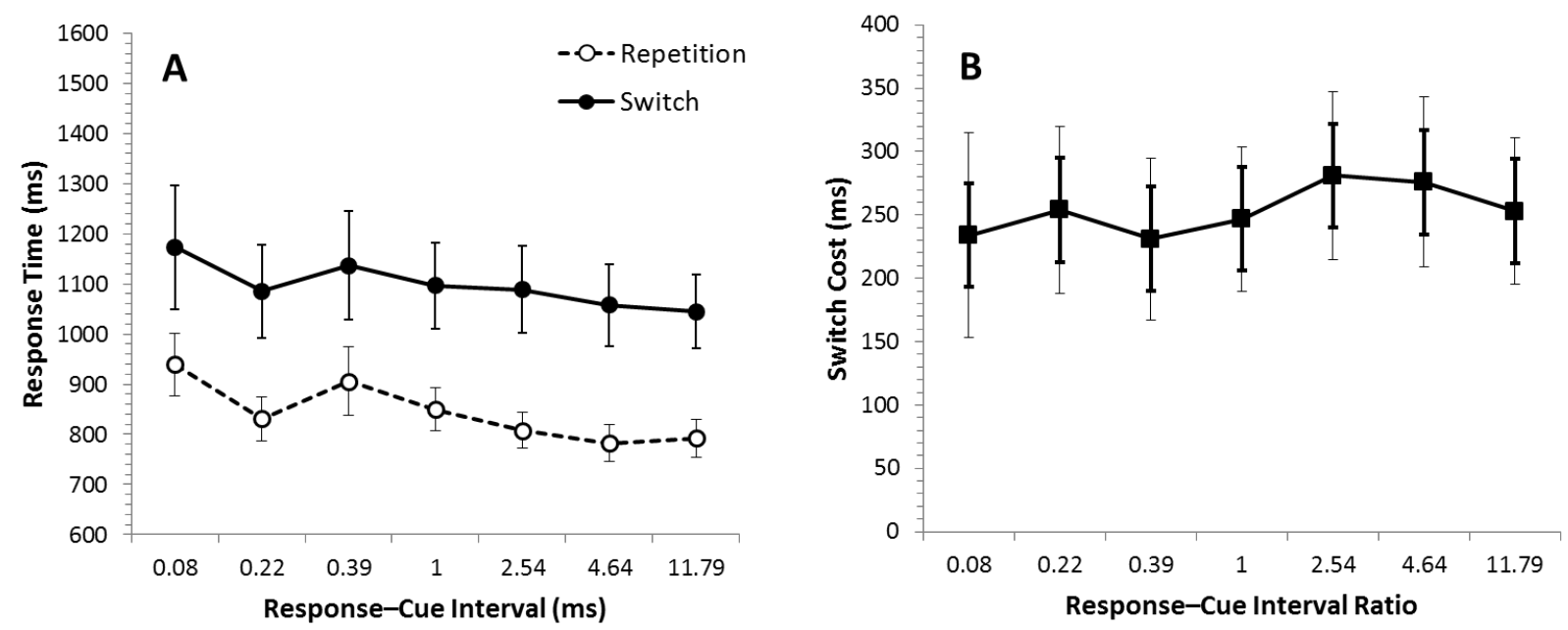

Figure 7. Data from Experiment 2. A. Mean response times (in milliseconds, ms) for task repetition and task switch trials as a function of response-cue interval ratio. Error bars denote $95 \%$ confidence intervals. B. Mean switch costs as a function of response-cue interval ratio. Bold error bars represent $+/-95 \%$ within-subject confidence intervals using the error term from the Sequence * RCI-ratio interaction (Loftus \& Masson, 1994); thin error bars represent standard $95 \%$ confidence intervals.

(Schneider \& Logan, 2005) assumes that short-term memory representations of the task cue decays passively. Thus, the interesting possibility arises that decay might affect perceptualbased aspects of task-sets (such as sensory-evidence from stimulus location in the paradigm reported below), whereas more "high-level" aspects of task-sets (such as biasing parameters to perform one task over the other; e.g., Logan \& Gordon, 2001) are not affected by decay. ${ }^{2}$ We have outlined this distinction at a very general level of description here, but we return to this issue in the General Discussion, and discuss how extant models of task switching might be able to formally account for this distinction.

Thus, for Experiment 3, we sought to investigate whether we could find decay-like patterns of data independent of temporal distinctiveness in a task switching paradigm with increased perceptual-judegement demands. To this end, we utilised the paradigm used by Meiran et al. (2000) which involves a perceptual decision from participants. Specifically, participants are presented with a $2 \times 2$ square grid, and on each trial a stimulus appears in any one of the four quadrants. The potential tasks are an up-down judgement-requiring a response as to whether the stimulus appears in the upper- or lower-two quadrants - and a left-right judgement - requiring a response as to whether the stimulus appears in the leftor right- two quadrants. Although higher-level task sets are still required in this task (such as representations of the stimulus-response mappings), it also has a strong perceptualcomponent (i.e., the tasks require perceptual judgements), so we are able to investigate whether we find decay of perceptual-settings.

\footnotetext{
${ }^{2}$ We thank Iring Koch for raising this possibility.
} 

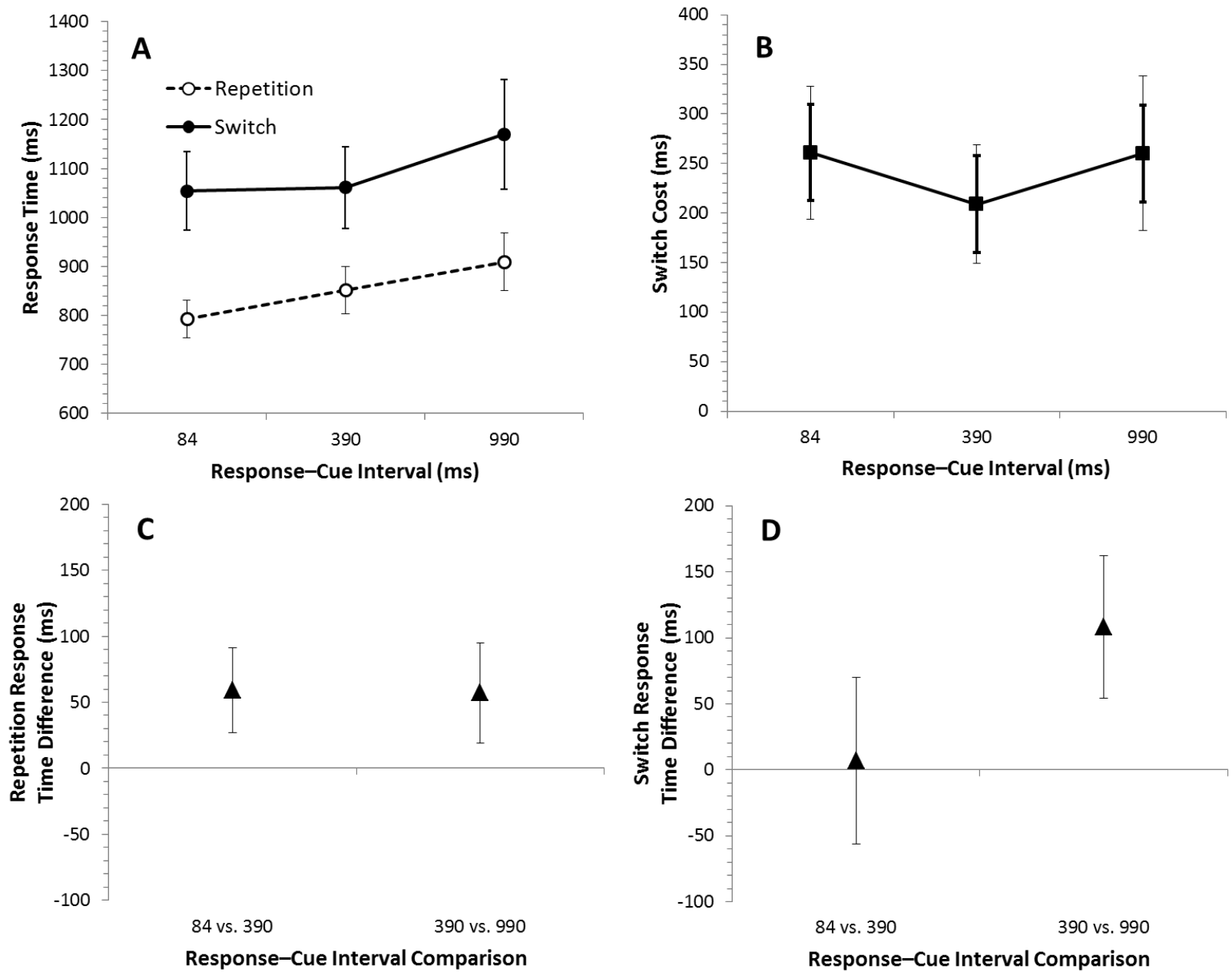

Figure 8. Data from Experiment 2 when the response-cue interval ratio is at unity. A. Mean response times (in milliseconds, ms) for task repetition and task switch trials as a function of response-cue interval. Error bars denote $95 \%$ confidence intervals. B. Mean switch costs as a function of response-cue interval. Bold error bars represent $+/-95 \%$ within-subject confidence intervals using the error term from the Sequence * RCI interaction (Loftus \& Masson, 1994); thin error bars represent standard 95\% confidence intervals. C. Pairwise comparisons showing mean differences as response-cue interval increases for task repetition trials, and D. for switch trials. Error bars denote $95 \%$ confidence intervals. 


\section{Experiment 3}

\section{Method}

Participants. 40 new participants were recruited from the same pool as Experiment 1; 8 additional participants were excluded due to session-wise accuracy below $90 \%$.

Stimuli \& Procedure. The same experimental apparatus was used as in Experiment 1 . On each trial, participants were presented with a blank $2 \times 2$ square black grid (total size $5 \mathrm{~cm} \times 5 \mathrm{~cm}$ ) on a grey background. After a variable response-cue interval, the task cues were presented. Cues were two small black arrows $(0.5 \mathrm{~cm}$ in height and width): for the 'up-down' task, there was one arrow pointing upwards, centered and on top of the grid, and one arrow pointing downwards, centered and on the bottom of the grid; for the left-right task, there was one arrow pointing leftwards, centered and on the left of the grid, and one arrow pointing rightwards, centered and on the right of the grid. The cue was presented for $100 \mathrm{~ms}$. After this time, a blue circle (1 $\mathrm{cm}$ diameter) appeared, centered within one of the four squares in the grid. In the up-down task, the participant was required to judge whether the stimulus appeared in the upper- or lower-half of the grid; in the left-right task, the participant was required to judge whether the stimulus appeared in the left- or the right-half of the grid. The response keys were the 7 and 3 keys on the numerical keypad of the keyboard. For all participants, the 7 key corresponded to eiter an "up" judgement or a "left" judgement; the 3 key corresponded to a "lower" judgement or a "right" judgement, depending on the currently relevant task. The stimulus and cues remained visible until participants made a response, at which time the stimulus and cues disappeared for a variable RCI, after which the next trial began. The RCIs used in this Experiment were 84, 190 990, and 1989ms. The Experiment consisted of 8 blocks of 96 trials, preceded by a practice block of 20 trials.

Design. The experiment manipulated two factors in a fully related design: Task sequence (switch vs. repetition) and $R C I(84 \mathrm{~ms}, 190 \mathrm{~ms}, 990 \mathrm{~ms}$, and $1989 \mathrm{~ms})$. The dependent variables throughout were again $\mathrm{RT}$ and error rates.

\section{Results}

Data trimming was identical to that of Experiment 1. Mean error rates are shown in Table 4, which showed fewer errors on task repetition trials than task switch trials $(-1.57 \%$ $[-2.08,-1.06]$. This difference appeared to remain consistent at extended RCIs: $-1.33 \%$ [$2.19,-0.46],-1.58 \%$ [-2.51, -0.66$],-1.51 \%$ [-2.64, -0.38], and $-1.86 \%$ [-2.84, -0.89] for RCIs $84,190,990$, and $1989 \mathrm{~ms}$ respectively.

The mean RTs for Experiment 3 are shown in Figure 9. As can be seen from Figure $9 \mathbf{A}$, there was a large switch cost at all RCIs, although the switch cost did reduce at longer RCIs (Figure $9 \mathbf{B}$ ). This reduction in switch cost was driven by gradual slowing of task repetition response time as RCI increased (Figure $9 \mathbf{C}$ ), and gradual facilitation of switch response time as RCI increased (Figure $9 \mathbf{D}$ ). Task repetition RTs did not slow between RCIs of $84 \mathrm{~ms}$ and $190 \mathrm{~ms}$, but there was slowing between RCIs of $190 \mathrm{~ms}-990 \mathrm{~ms}$ and $990 \mathrm{~ms}-$ $1989 \mathrm{~ms}$; for switch RTs, there was facilitation between RCIs of $84 \mathrm{~ms}-190 \mathrm{~ms}$, and smaller, less consistent, facilitation between RCIs of $190 \mathrm{~ms}-990 \mathrm{~ms}$ and $990 \mathrm{~ms}-1989 \mathrm{~ms}$ with the CIs of the differences including zero. Note that this pattern of data-slowing repetition RTs and speeding switch RTs - is prototypical of a decay account. 

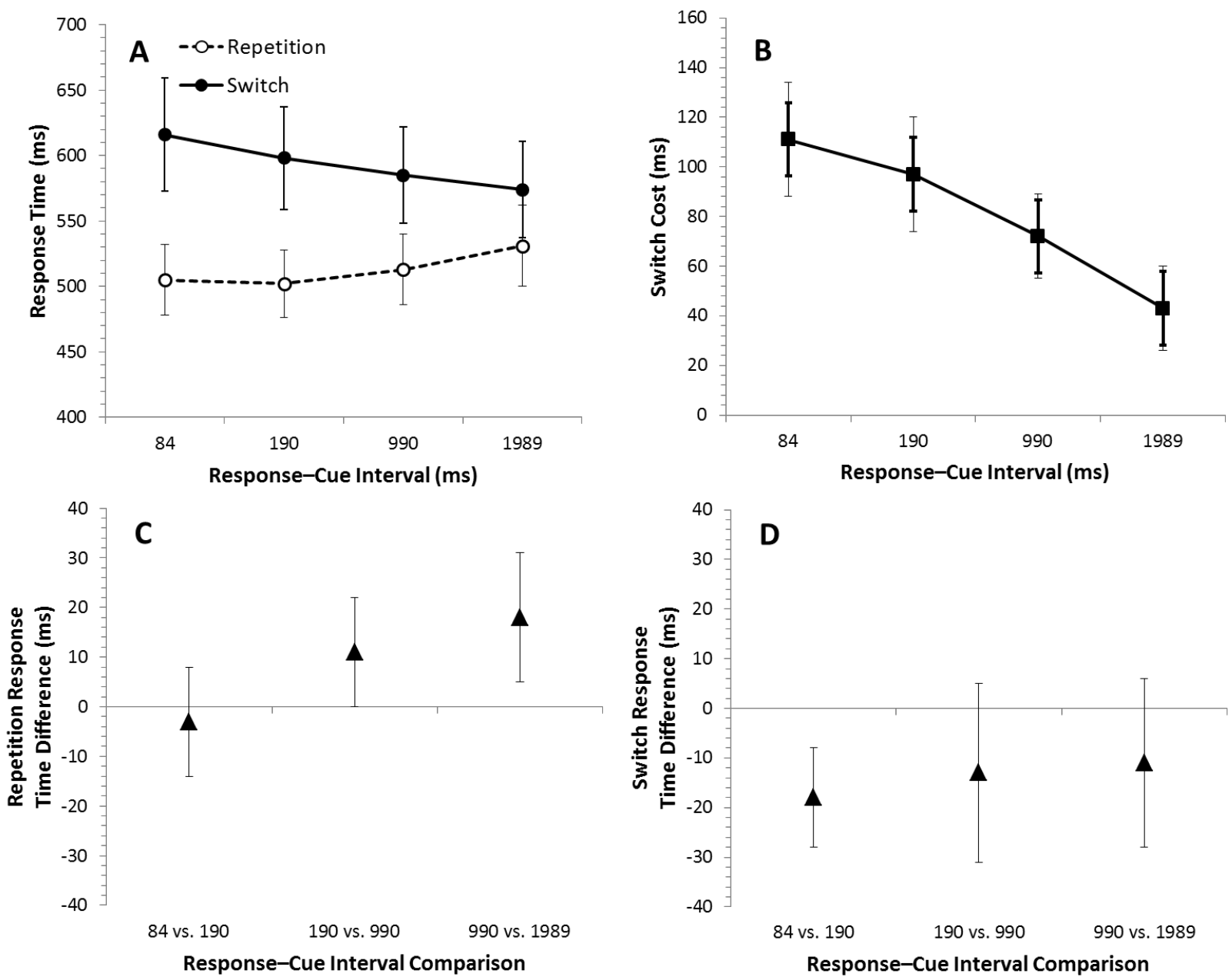

Figure 9. Data from Experiment 3. A. Mean response times (in milliseconds, ms) for task repetition and task switch trials as a function of response-cue interval. Error bars denote $95 \%$ confidence intervals. B. Mean switch costs as a function of response-cue interval. Bold error bars represent +/- 95\% within-subject confidence intervals using the error term from the Sequence * RCI interaction (Loftus \& Masson, 1994); thin error bars represent standard 95\% confidence intervals. C. Pairwise comparisons showing mean differences as responsecue interval increases for task repetition trials, and D. for switch trials. Error bars denote $95 \%$ confidence intervals. 
Table 4

Mean error rates (\%) for Experiment 3 with 95\% confidence intervals in square brackets, for both the global analysis and the RCI-ratio unity analysis.

\begin{tabular}{ccccc}
\hline & \multicolumn{2}{c}{ Global Analysis } & \multicolumn{2}{c}{ Unity Analysis } \\
\hline RCI & Repetition & Switch & Repetition & Switch \\
\hline 84 & $2.65[1.95,3.35]$ & $3.97[2.98,4.96]$ & $3.30[1.61,4.99]$ & $4.24[2.78,5.70]$ \\
190 & $2.95[2.08,3.82]$ & $4.54[3.63,5.45]$ & $3.68[2.01,5.35]$ & $4.10[2.49,5.71]$ \\
990 & $3.57[2.54,4.60]$ & $5.08[3.97,6.19]$ & $5.72[3.86,7.61]$ & $6.04[4.32,7.76]$ \\
1989 & $2.61[1.77,3.45]$ & $4.47[3.41,5.53]$ & $2.83[1.46,4.20]$ & $4.46[2.82,6.10]$ \\
\hline
\end{tabular}
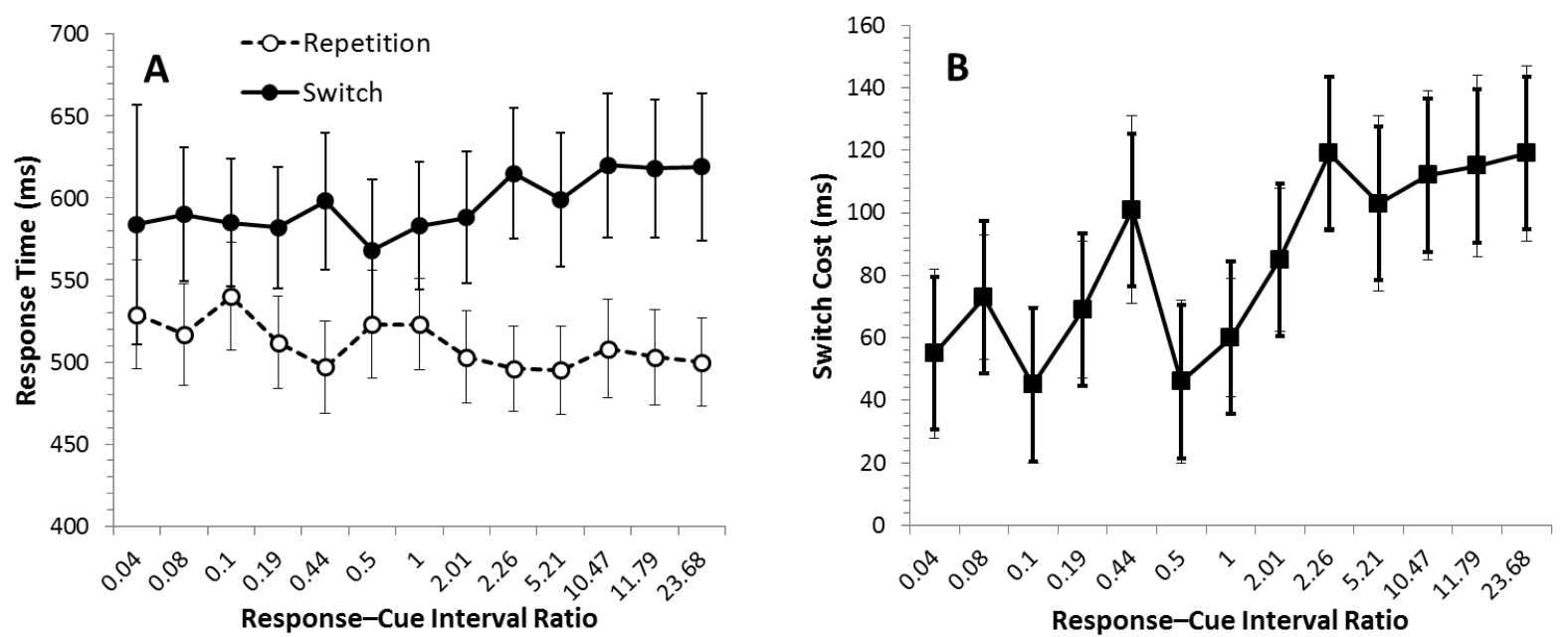

Figure 10. Data from Experiment 3. A. Mean response times (in milliseconds, ms) for task repetition and task switch trials as a function of response-cue interval ratio. Error bars denote $95 \%$ confidence intervals. B. Mean switch costs as a function of response-cue interval ratio. Bold error bars represent $+/-95 \%$ within-subject confidence intervals using the error term from the Sequence* RCI-ratio interaction (Loftus \& Masson, 1994); thin error bars represent standard $95 \%$ confidence intervals.

The RTs as a function of RCI-ratio can be seen in Figure 10. Unlike the previous experiments, there was no clear effect of RCI-ratio on repetition RT: RTs were $529 \mathrm{~ms}$ [496, $562]$ at the 0.04 ratio, and only 500ms [473, 527] at the 23.68 ratio. However, switch RTs did tend to slow as RCI-ratio increased. The net effect was a general increase in the switch cost as RCI-ratio increased (Figure $10 \mathrm{~B}$ ).

Ratio-unity analysis. Mean RCI-ratio unity error rates are shown in Table 4, which showed fewer errors on task repetition trials than task switch trials $(-0.83 \%[-1.63$, -0.03]. There was no clear change in this difference as RCI extended, as zero was a credible estimate for the difference at each RCI: $-0.95 \%[-3.35,1.46],-0.42 \%[-2.06,1.23],-0.32 \%$ $[-2.15,1.51]$, and $-1.63 \%$ [-3.46, 0.19] for RCIs 84, 190, 990, and 1989ms respectively.

The RT data when the RCI-ratio is at unity is shown in Figure 11 A.

There was no clear effect of RCI on task repetition RT (Figure $11 \mathbf{C}$ ), in contrast to Experiments 1 and 2, and in fact showed slight facilitation between RCIs of $84-190 \mathrm{~ms}$; 
between RCIs of 190-990ms and 990-1989ms, there was no clear slowing of RT, contra the decay account. A decay account should predict slowing of repetition RTs across all RCIs, so the slight speeding of repetition RTs between RCIs of $84 \mathrm{~ms}-190 \mathrm{~ms}$ is not accountable for by a decay process. The slowing of repetition RTs between RCIs of 190-990ms and 990ms$1989 \mathrm{~ms}$ was very small (6ms and 11ms, respectively), and not credibly different from zero. Thus, the repetition RT provides no clear evidence for a decay process.

However, switch RTs (Figure 11 D) did tend to speed as RCI increased, although again the CIs of these differences included zero at each RCI comparison. However, the combined effect of these opposing trends - slight speeding of switch RT and negligible slowing of repetition RT - led to a clear and consistent reduction in the switch cost as the RCI increased (Figure 11 B).

\section{Discussion}

In this experiment, we sought evidence of decay in an experimental paradigm that emphasises perceptual judgements more than in Experiments 1 and 2, examining whether perceptual representations are subject to decay. We find some evidence (albeit rather weak) for decay when the RCI-ratio is at unity. We say weak because there was no clear slowing for repetition RT and no clear facilitation to RT between each successive RCI, but the combined opposing trends in the data led to a consistent reduction in switch cost as RCI increased, predicted by a decay account. Thus, this experiment - together with the findings of Horoufchin et al. (2011a, Experiment 4)-provides tentative evidence that perceptual representations might be subject to decay.

The effect of RCI-ratio was not as pronounced as in either of the first two experiments, or in the original data of (Horoufchin et al., 2011a). This reduced effect of TD could be due to the current paradigm emphasising perceptual representations, which might be subject to decay rather than temporal distinctiveness. We return to this discussion in the General Discussion.

\section{General Discussion}

The present work has explored whether a decay account can explain the pattern of data attributed to temporal distinctiveness. The TD account suggests that RCI effects in task switching are not caused by decay of task-sets in memory (cf., Meiran et al., 2000). Rather, RCI effects arise in task switching due to the sequential variation in RCI, which affects the distinctiveness of a target task in episodic memory, affecting repetition priming. This interference-based explanation (Brown et al., 2007) of the data provides an interesting hypothesis in contrast to extant models of task switching (Altmann \& Gray, 2008) that assume memory for tasks decay as a function of time. In the model of Altmann and Gray (2008) this decay is considered functional, in that it allows the system not to be dominated by recently performed actions; without decay, tasks will clutter episodic memory making switching to new tasks challenging. As decay is central to this successful model, it is important to assess whether the RCI-ratio effect can be explained by decay processes.

The contributions of this work are (1) to demonstrate that a confound exists between RCI-ratio and the current RCI; as such, a decay model is able reproduce data attributed to TD (Appendix B); (2) to demonstrate that the critical test of the decay vs. TD hypothesis 

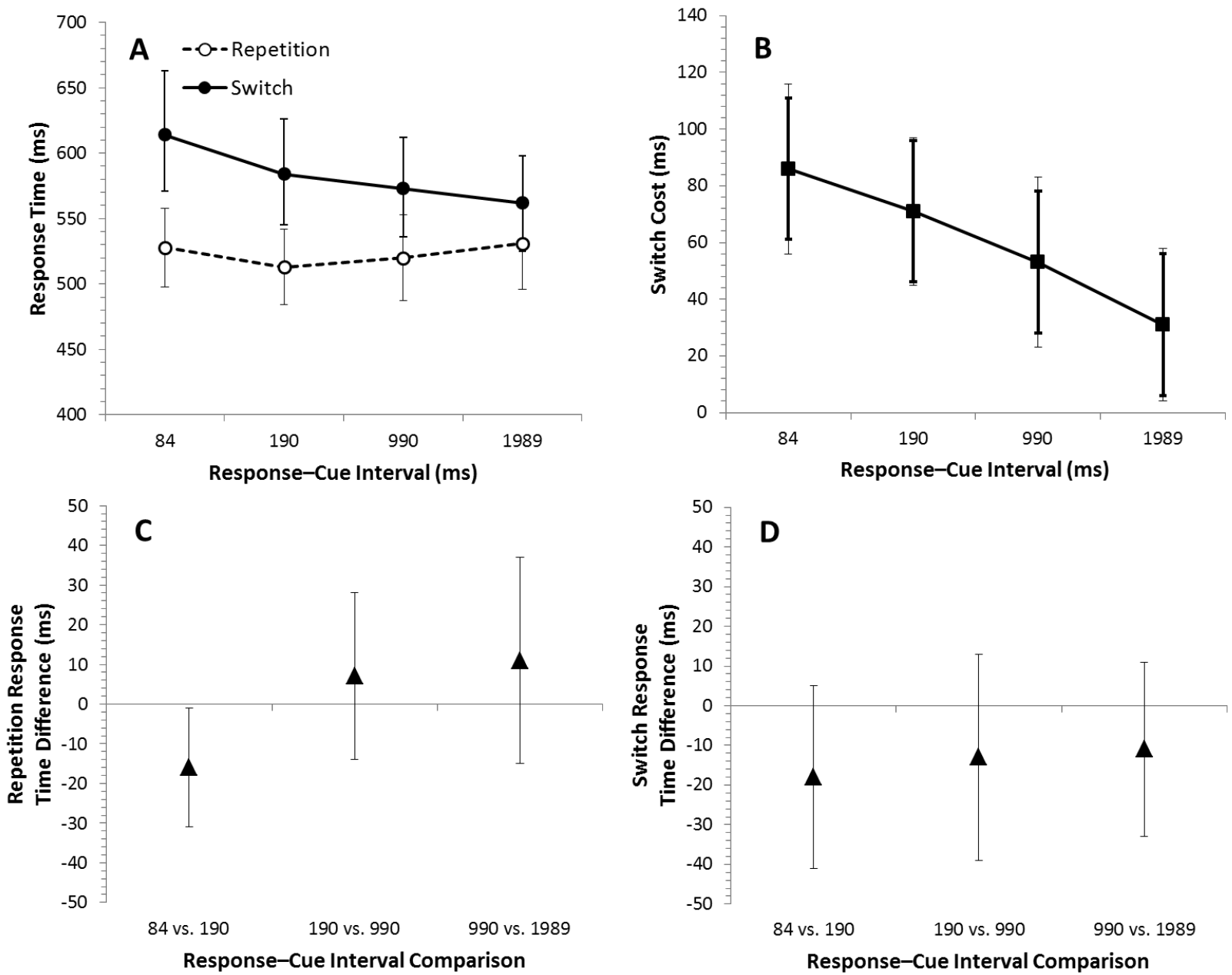

Figure 11. Data from Experiment 3 when the response-cue interval ratio is at unity. A. Mean response times (in milliseconds, ms) for task repetition and task switch trials as a function of response-cue interval. Error bars denote $95 \%$ confidence intervals. B. Mean switch costs as a function of response-cue interval. Bold error bars represent $+/-95 \%$ within-subject confidence intervals using the error term from the Sequence * RCI interaction (Loftus \& Masson, 1994); thin error bars represent standard 95\% confidence intervals. C. Pairwise comparisons showing mean differences as response-cue interval increases for task repetition trials, and D. for switch trials. Error bars denote $95 \%$ confidence intervals. 
is when the RCI-ratio is at unity; this realisation arose from a formal implementation of the theory of Horoufchin et al. (2011a; see Appendix A); (3) to show that tests of this prediction provide little support for decay processes (Experiments 1 and 2), except when the perceptual demands of the task are increased (Experiment 3). Taken together, we suggest the evidence for decay of higher-level task-sets in this study is weak at best. We discuss these contributions in turn below. In addition, we discuss how the formal models of these accounts (Appendix A and B) might be extended to aid future research in disentangling the decay and TD accounts of RCI effects in task switching.

\section{Decay vs. Temporal Distinctiveness}

The present work's primary contribution is to highlight that a decay account can reproduce the pattern of data attributed to temporal distinctiveness (Figure 1). This is because a confound exists between RCI-ratio and the current RCI. As such, there is a degree of mimicry between the accounts, in that they can both explain the data with different theoretical underpinnings. We have demonstrated that to differentiate between the two accounts one must analyse data when the RCI-ratio is at unity; it is this scenario where one can examine potential effects of decay independent of interference effects (and indeed, this is the strategy followed by Horoufchin et al., 2011a, in their later experiments; see below). As the confound between RCI-ratio and RCI is strong (see Table 1), it is essential that researchers take this into account when designing studies addressing decay vs. TD in task switching.

We tested the strong prediction of decay-slowing repetition RTs and speeding switch RTs with increasing RCI when the ratio is at unity - in re-analysis of the data from Horoufchin et al. (2011a), which provided some support for this account. However, in Experiments 1 and 2, the decay prediction was not supported as we reported slowing of RTs for repetition and switch trials, suggesting that the slowing is not caused by a task-set decay process (Meiran et al., 2000). It is not clear at present what is being slowed for switch trials as RCI increases, but it might reflect more general, task-independent effects. For example, (Altmann, 2005) suggested that RCI effects might be caused by variation in phasic alertness with RCI (see also Meiran, 2005); such variance in alertness would be taskindependent and would thus affect task repetition and task switch trials equally. Thus, the results of Experiments 1 and 2 provide falsification of the decay hypothesis; however, one might also ask whether the same data falsifies the TD account (which predicts no change in repetition RT as RCI increases at RCI-ratio unity). We suggest that an extension of the TD model can explain this data, and return to the discussion of this issue in the section "Can TD explain increasing RTs with increasing RCI?".

The finding of increasing RTs with increasing RCIs when the RCI-ratio is held at unity is an interesting finding, but stands in direct contrast to data reported in Experiment 2 and 3 of Horoufchin et al. (2011a; see also Horoufchin et al., 2011b). Using two RCIs, these authors compared performance between long and short RCIs as a function of whether the RCI value changed from the previous trial (i.e., 100-1,000 \& 1,000-100) or remained unchanged (i.e., 100-100 \& 1,000-1,000). They found repetition RTs slowed at longer RCIs only when the RCI had changed from the previous trial (i.e., 100-1,000), in line with the TD account. When the RCI did not change - i.e., when the RCI-ratio was at unity - they reported no slowing of repetition RT with increasing RCI. 
It is not clear why there should be slowing for repetition and switch RTs with extended RCIs when the RCI-ratio is at unity in the current data but not in the latter experiments of Horoufchin et al. (2011b). It is important to note again that the slowing for repetition trials and speeding of switch trials with extended RCI was found in the re-analysis of Experiment 1 of Horoufchin et al.. It is possible that the number of RCIs being manipulated can have some effect on the data, but it is not clear why this should either affect time-based decay processes or temporal distinctiveness. However, this type of analysis clearly avoids the issue of the dependency between RCI and RCI-ratio, and thus remains a very hard set of data for decay accounts to explain.

Effect of RCI and RCI-ratio on Switch Trials. The finding of slowing switch RTs with increasing RCI in Experiments 1 and 2 appears quite atypical for the task switching literature (Horoufchin et al., 2011a, 2011b). The atypicality was a concern in Experiment 1, but the effect replicated in Experiment 2 using quite different task demands. However, other authors have presented slowing for switch trials, too; for example, Experiment 2 of Sohn and Anderson (2001) showed extensive slowing for switch and repetition RT at RCIs up to 5 seconds (although the slowing was greater for repetition trials). In addition, Koch and Lawo (2014) reported slowing for repetition and switch RTs in a newly-developed auditory version of the task switching paradigm. It is not clear what is driving this inconsistent effect of RCI on task switch RT across studies.

Although Experiments 1 and 2 reported here largely replicated the RCI-ratio effects reported by (Horoufchin et al., 2011a), we additionally report an effect of RCI-ratio on switch RTs, although the effect does appear stronger for repetition trials. Note that neither the verbal theory of Horoufchin et al. (2011a) or the SIMPLE model in Appendix A speak to the effects of RCI-ratio on switch trials, so this is an essential area to explore in future research (see the section "Formalising Decay and $T D$ " for more on this).

Potential Decay of Perceptual Representations?. In Experiment 3, we pursued the idea first proposed by Horoufchin et al. (2011a) in their Experiment 4 that perceptual representations might be subject to decay (see also Koch \& Lawo, 2014). To this end, we utilised the paradigm of Meiran et al. (2000) which is based on perceptual judgements (up-down vs. left-right judgements of visual stimuli), in contrast to Experiments 1 and 2 which utilised more "central", memory-driven, tasks. In this experiment, we presented some evidence supporting decay in the form of a reduction of switch cost with extended RCI when the RCI-ratio was at unity, driven by trends for repetition RTs to slow as RCI increased, and for task switch RTs to become facilitated as RCI increased. It should be noted, though, that this pattern of data differs to that reported by Meiran et al. (2000); we return to this point in the section "On Replicability of RCI Effects" later in the General Discussion. However, the findings we report supports the proposal of Horoufchin et al. (2011a) that decay might occur when the task has higher perceptual demands rather than more demand for memory retrieval of task-sets from episodic memory.

Although this idea is certainly in need of formal elaboration and empirical validation, it appears consistent with evidence that visual short-term memory contents appear subject to decay independent of interference effects (Ricker, Spiegel, \& Cowan, in press). However, caution must be taken comparing performance in a task switching context and performance in a pure memory task. It is also not clear how decay of perceptual representations being subject to decay yet higher-level task-sets being subject to temporal distinctiveness can be 
explained by extant models of task switching.

\section{Formalising Decay and TD}

It is essential for future work to elaborate the modelling presented in Appendices A and $\mathrm{B}$ in order to disentangle the decay and TD hypotheses. This work is important as verbal theories alone are too flexible, and as such are hard to falsify (Farrell \& Lewandowsky, 2010). This problem is compounded when attempting to differentiate between competing accounts which are both equally able to qualitatively explain extant data, as is the case in the current study.

The models in Appendix A and B were developed merely to produce broad quantitative predictions of the TD and the decay hypotheses (cf., verbal theories) to demonstrate the mimicry between the two accounts, but they are -in their present form-too simple to be utilised for rigorous competitive model comparison which is essential to determine which model provides a better account of extant data. The models certainly serve as a good starting point for this elaboration, but need to be developed further by adding a true RT module to each-likely in the form of a diffusion process, a successful model of choice RT (e.g., Wagenmakers, 2009). Adding a mechanism to each model capable of producing whole RT distributions will allow researchers to utilise maximum likelihood estimation of parmaeter values, and thus engage in competitive model comparison using statistical model-comparison techniques (such as Akaike's Information Criterion; e.g., Lewandowsky \& Farrell, 2010). Rigorous statistical model comparison of decay and TD models is an essential next stage in this research programme to aid our understanding of the dynamics of task-set representations.

The current models are also rather abstract in the sense that they do not at present specifiy what is retrieved in the TD model and what is decaying in the decay model. This abstractness was sufficient for our current demonstration - and would arguably be sufficient to engage in the competitive model comparison stated above - as the abstractness allows us to explore the general dynamics of a decay and TD model without committing ourselves to a specific theoretical stance as to how tasks and task-sets are represented and controlled. A welcome advance, however, would be to formalise what aspects of the task-set are being retrieved / are decaying. Such specification would also have to address performance on switch trials, which neither model currently do. Indeed, all experiments have demonstrated effects of RCI and/or RCI-ratio on switch trials as well as repetition trials, but so far theoretical accounts are relatievly silent on the effect of RCI on switch trials. For example, verbal decay accounts (e.g., Meiran et al., 2000) predict speeding of switch RT as RCI increases due to decaying activation of irrelevant tasks in memory, which induce less proactive interference. Currently, though, the decay account does not specify in any formal detail what level of the task set is generating proactive interference, or how this interference is resolved when it is sufficiently high.

One possibility in this endeavour would be to examine RCI effects in extant models of task switching, and to compare model architectures which assume decay and ones which do not. The formal model of Schneider and Logan (2005), for example, is a promising candidate as it is capable of produing a plethora of empirical task switching results, and has the advantage that the representations that guide behaviour are explicitly stated in the model. For example, the model assumes that task performance is achieved by encoding 
the task cue, encoding the stimulus, and using both as a compound-cue to probe longterm memory for the correct response. Cues and stimuli uniquely provide varying degrees of evidence for response categories (e.g., the cue "ODD" provides strong evidence for the response category "odd", but weak evidence for the category "lower than 5"); the evidence from the cue and stimulus is combined muliplicatively to form final evidence values for each response category, which determine the speed and accuracy of response selection.

In the framework of this model, one might be able to explore the effect of decay and TD by having the model "remember" aspects of the task-set used on previous trials. For example, from trial-to-trial, the final evidence values used on prior trials could decay (decay hypothesis), or have a certain probability of being retrieved again (TD hypothesis) on the current trial (which might facilitate performance). Within this framework, one might also be able to explore the possible dissociation between decay/TD of perceptual/"higher-level" representations, respectively. For example, the model also has a parameter that reflects the participant's bias to perform one of two tasks (see also Logan \& Gordon, 2001). This bias parameter can increase the evidence for certain response categories (e.g., "odd" and "even" if the system is biased to perform the "parity" task), and is formed from the participant's homunculus, rather than by perceptual input (unlike the evidence values from cues and stimuli). Although this parameter is effectively removed from the models of Schneider and colleagues (as perceptual representations of cues and stimuli alone are sufficient to drive performance), it can be introduced to explore the idea that perceptual representations decay, but task bias does not (Experiment 3; see also Horoufchin et al., 2011a). Of course, this is just one possibility to explore.

Can TD Explain increasing RTs with increasing RCI?. Further formalisation of the TD hypothesis might also allow us to address empirical effects that appear inconsistent with this account. For example, in Experiments 1 and 2 reported here, and the re-analysis of Horoufchin et al. (2011b), we found an effect of RCI on repetition trials when the RCI-ratio was at unity. Recall that the TD account - as currently implemented - does not predict this effect (Appendix A), and one might suggest that this pattern falsifies the TD account. However, it is possible that this increase in repetition RT can be accomodated by an extended TD account, when RCIs beyond that of the current- and previous-trial are considered $^{3}$.

For example, consider the presentation of four consecutive tasks: D-C-B-A, where ' $\mathrm{A}$ ' represents the cue presentation for the current task, and ' $\mathrm{B}$ ' is the target task which is to be retrieved. Now consider the following RCI sequences between each task:

(1) D-100-C-2000-B-2000-A (2) D-2000-C-2000-B-2000-A.

The temporal distinctiveness account in its current form would predict the same distinctiveness of task $\mathrm{B}$ in both examples as it only considers the RCI from n-2 (i.e., between $\mathrm{C}-\mathrm{B}$ ) and $\mathrm{n}-1$ (between $\mathrm{B}-\mathrm{A}$ ), and in both examples the RCI-ratio is at unity. However, when considering the RCI from n-3 (i.e., between $\mathrm{D}-\mathrm{C}$ ), the distinctiveness of the target task can change. In the first example above, task B is more clustered in psychological space than it is in the second example, because it has an additional crowded neighbour from task D. The additional crowding reduces the probability of retrieving task B due to increased confusibility.

${ }^{3}$ We are extremely grateful to an anonymous reviewer for encouraging us to explore this issue. 
Thus, $\mathrm{n}-3$ can influence current-trial performance if it is not taken into consideration. But, can it explain the increase in repetition RT with increasing RCI of the current trial when the RCI-ratio is at unity? It seems that this is certainly possible (see Brown et al., 2007, for a similar discussion of an apparent effect of time when prior trials are not considered in the context of a memory experiment); in Appendix $\mathrm{C}$ we present example calculations using the formal TD model in Appendix A. We summarise the main points here.

The calculations show that the influence of $n-3$ 's RCI on current trial performance reduces as the $\mathrm{n}-2$ and $\mathrm{n}-1$ RCIs reduce (see Table $\mathrm{C} 1$ ); that is, $\mathrm{n}-3$ 's RCI only affects retrieval probability of the desired task when the current- and previous RCI is long. This is due to the log compression of memory traces in SIMPLE as time progresses (see Appendix A). This means that with shorter RCIs at $n-2$ and $n-1$, the target trace will still be relatively isolated in psychological space, and as such, clustering of the trace from n-3 has less effect. In contrast, when the RCI at n-2 and n-1 are longer, due to the log compression the trace from $n-1$ will already be quite clusterd in psychological space, meaning the additional clustering from n-3 can affect retrieval probability more strongly.

The net effect of the decreasing effect of $n-3$ RCI as the $n-2$ and $n-1$ RCI increase is that the average distinctiveness of traces at $n-1$ decrease as the current trial's RCI increases. For example, the distinctiveness (which is proportional to retrieval probability) of the target trace when the current RCI is $2000 \mathrm{~ms}$ ranges from 0.896 to 0.932 , in contrast to the distinctiveness values when the current RCI is $100 \mathrm{~ms}$ which range from 0.932 to 0.942 (see final column in Table C1). The average distinctiveness of the target trace thus increases as current RCI increases: $0.912,0.919,0.934$, and 0.939 for RCIs of 100, 200, 1000, and $2000 \mathrm{~ms}$ respectively. As mean RT increases as average retrieval probability decreases, the TD model can indeed produce increasing RTs with increasing RCI, even though the RCI-ratio is at unity.

\section{On the Replicability of RCI Effects}

As a brief note, we wish to discuss the apparent lack of replicability of RCI effects in task switching. Replication is an essential aspect of any research programme interested in building a cumulative science (Brandt et al., 2014), and replicability of empirical results in psychological science is starting to gain interest in the experimental community (Open Science Collaboration, 2012)

In this paper, we have conducted two relatively close replications of extant work (Experiment 1 was a close replication of Horoufchin et al., 2011a, and Experiment 3 was a close replication of Meiran et al., 2000). In both cases, we report different results to those reported by the original authors. Whilst the TD effects replicated relatively well in Experiment 1, we had difficulty replicating the key effect predicted by a decay account (which we found was evident in the Horoufchin et al., 2011a data): Slowing of repetition RT and speeding of switch RT with increasing RCI. In Experiment 3, we broadly replicated the key effect of a reduction of switch cost with extended RCI reported by Meiran et al., but in our case this was mostly driven by a reduction of switch RT; repetition RT did not appear affected by RCI.

These findings - as well as providing a certain challenge to the strong predictions of a decay account-might speak more widely to a lack of replicability of RCI effects in 
task switching. Thus, researchers interested in understanding whether task-sets are subject to decay or are lost due to interference might wish to initially engage in a programme of research addressing the replicability of empirical effects attributed to decay. Besides the RCI effect, there also exists evidence of decay in the form of within-run slowing - the finding of progressively slowing RTs as one performs a run of the same task, thought due to the decay of that task's representation (Altmann \& Gray, 2008) - and RCI effects on measures of inhibition in task switching (Gade \& Koch, 2005; Grange \& Houghton, 2009). Only once the replicability of key effects attributed to decay can be established can a research programme continute to systematically assess the relative evidence for decay and interference of task-set representations.

\section{Conclusion}

The question of whether task-set representations decay as a function of time or are lost due to interference has strong implications for current models of cognitive control in task switching (Grange \& Houghton, 2014). This question therefore remains an important area for researchers interested in task control. In this paper we have explored a potential role for decay in explaining patterns of data attributed to temporal distinctiveness; however, we find no strong evidence in support of decay, except weak evidence for decay of perceptual settings. We have also discussed how the TD hypothesis - as currently implemented - is challenged by the data reported here. However, we have shown that a simple extension to the TD account can explain this apparent discrepancy. Such discussion has highlighted the importance of formalising these hypotheses into computational models to explore their quantitative predictions. There additionally remains the possibility to explore that decay and TD - together - give rise to the complex pattern of RCI effects in task switching (e.g., Altmann \& Shunn, 2012). Such work is essential to understand the dynamics of task-set representations that guide behaviour.

\section{References}

Allport, A., Styles, E., \& Hsieh, S. (1994). Shifting intentional set: Exploring the dynamic control of tasks. In C. Ulmita \& M. Moscovitch (Eds.), Consciousness and Nonconscious Information Processing: Attention and Performance XV (p. 421-452). Cambridge, MA: MIT Press.

Altmann, E. M. (2005). Repetition priming in task switching: Do the benefits dissipate? Psychonomic Bulletin \& Review, 12, 535-540.

Altmann, E. M. (2009). Evidence for temporal decay in short-term episodic memory. Trends in Cognitive Science, 13, 279-279.

Altmann, E. M., \& Gray, W. D. (2008). An integrated model of cognitive control in task switching. Psychological Review, 115, 602-639.

Altmann, E. M., \& Shunn, C. D. (2012). Decay versus interference: A new look at an old interaction. , 23, 1435-1437.

Anderson, J. R. (2007). How can the human mind occur in the physical universe? Oxford: University Press.

Brandt, M. J., IJzermanm, H., Dijksterhuis, A., Farach, F., Geller, J., Giner-Sorolla, R., ... van t' Veer, A. (2014). The replication recipe: What makes for a convincing replication? Journal of Experimental Social Psychology, 50, 217-224. doi: 10.1016/j.jesp.2013.10.005

Brown, G. D. A., Neath, I., \& Chater, N. (2007). A temporal ratio model of memory. Psychological Review, 114, 539-576. 
Cumming, G. (2014). The new statistics: Why and how. Psychological Science, 25, 7-29.

Farrell, S., \& Lewandowsky, S. (2010). Computational models as aids to better reasoning in psychology. Current Directions in Psychological Science, 19, 329-335.

Gade, M., \& Koch, I. (2005). Linking inhibition to activation in the control of task sequences. Psychonomic Bulletin \& Review, 12, 530-534.

Grange, J. A., \& Houghton, G. (2009). Temporal cue-target overlap is not essential for backward inhibition in task switching. Quarterly Journal of Experimental Psychology, 62, 2069-2080.

Grange, J. A., \& Houghton, G. (2014). Models of cognitive control in task switching. In J. A. Grange \& G. Houghton (Eds.), Task switching and cognitive control. New York, NY: Oxford University press.

Grange, J. A., Juvina, I., \& Houghton, G. (2013). On costs and benefits of n-2 repetitions in task switching: Towards a behavioural marker of cognitive inhibition. Psychological Research, 77, $211-222$.

Horoufchin, H., Philipp, A. M., \& Koch, I. (2011a). The dissipating task-repetition benefit in cued task switching: Task-set decay or temporal distinctiveness? Journal of Experimental Psychology: Human Perception and Performance, 37, 455-472.

Horoufchin, H., Philipp, A. M., \& Koch, I. (2011b). Temporal distinctiveness and repetition benefits in task switching: Disentangling stimulus-related and response-related contributions. Quarterly Journal of Experimental Psychology, 64, 434-446.

Kiesel, A., Steinhauser, M., Wendt, M., Falkstein, M., Jost, K., Philipp, A., \& Koch, I. (2010). Control and interference in task switching - a review. Psychological Bulletin, 136, 849-874.

Koch, I., \& Lawo, V. (2014). Exploring temporal dissipation of attention settings in auditory task switching. Attention Perception and Psychphysics, 76, 73-80.

Lewandowsky, S., \& Farrell, S. (2010). Computational modeling in cognition: Principles and practice. Thousand Oaks, CA: Sage.

Lewandowsky, S., Oberauer, K., \& Brown, G. D. A. (2009a). No temporal decay in verbal short-term memory. Trends in Cognitive Science, 13, 120-126.

Lewandowsky, S., Oberauer, K., \& Brown, G. D. A. (2009b). Response to Altmann: Adaptive forgetting by decay or removal of STM contents? Trends in Cognitive Science, 13, 280-281.

Loftus, G. R., \& Masson, M. E. J. (1994). Using confidence intervals in within-subject designs. Psychonomic Bulletin 83 Review, 1, 476-490.

Logan, G. D. (1988). Toward an instance theory of automization. Psychological Review, 95, 492-527.

Logan, G. D., \& Bundesen, C. (2003). Clever homunculus: is there an endogenous act of control in the explicit task-cuing procedure? Journal of Experimental Psychology: Human Perception and Performance, 29, 575-599.

Logan, G. D., \& Gordon, R. D. (2001). Executive control of visual attention in dual-task situations. Psychological Review, 108, 393-434.

Mayr, U., \& Kliegl, R. (2003). Differential effects of cue changes and task changes on task-set selection costs. Journal of Experimental Psychology: Learning, Memory, and Cognition, 29, $362-372$.

Meiran, N. (2005). Task rule-congruency and simon-like effects in switching between spatial tasks. Quarterly Journal of Experimental Psychology: A, 58, 1023-1041.

Meiran, N., Chorev, Z., \& Sapir, A. (2000). Component processes in task switching. Cognitive Psychology, 41, 211-253.

Neath, I., \& Brown, G. D. A. (2012). Arguments against memory trace decay: A SIMPLE account of Baddeley \& Scott. Frontiers in Cognition, 3, 35. doi: 10.3389/fpsyg.2012.00035

Open Science Collaboration. (2012). An open, large-scale, collaborative effort to estimate the reproducibility of psychological science. Perspectives on Psychological Science, 7, 657-670. doi: $10.1177 / 1745691612462588$

Ricker, T. J., Spiegel, L. R., \& Cowan, N. (in press). Time-based loss in visual short-term memory is from trace decay, not temporal distinctiveness. Journal of Experimental Psychology: Learning, 
Memory, and Cognition.

Schneider, D. W., \& Logan, G. D. (2005). Modeling task switching without switching tasks: A shortterm priming account of explicitly cued performance. Journal of Experimental Psychology: General, 134, 343-367.

Sohn, M.-H., \& Anderson, J. R. (2001). Tak preparation and task repetition: Two-component model of task switching. Journal of Experimental Psychology: General, 130, 764-778.

Vandierendonck, A., Liefooghe, B., \& Verbruggen, F. (2010). Task switching: interplay of reconfiguration and interference. Psychological Bulletin, 136, 601-626.

Wagenmakers, E.-J. (2009). Methodological and empirical developments for the Ratcliff diffusion model of response times and accuracy. European Journal of Cognitive Psychology, 21, 641671.

Appendix A

Modelling Temporal Distinctiveness with SIMPLE

The current section attempts to formalise the verbal theory of Horoufchin et al. (2011a) who explained performance on task repetition trials as benefiting from episodic retrieval of the previous trial's episodic trace when temporal distinctiveness is high; as the desired task is already active in working memory (Mayr \& Kliegl, 2003), any such episodic retrieval would prime responding further, leading to fast RTs. On the contrary, when episodic retrieval fails, less/no repetition priming is available, leading to relatively slower RTs. This theory assumes no time-based decay in task switching.

Thus, this hypothesis proposes that response times for task repetition trials consists of either successful episodic retrieval $\left(R T_{\text {Retrieved }}\right)$ or unsuccessful episodic retrieval $\left(R T_{\text {NotRetrieved }}\right)$, uniquely determined by the TD of the target task's episodic trace at $\mathrm{n}-1$. Another interpretation of the TD hypothesis is that the task cue triggers a race between two task-set "activation" processes: one process is a slow, algorithmic, activation process based on the learned association between the cue and the relevant task-set, and one is a fast episodic-retrieval process whereby the cue activates all previous instances of tasks in long-term memory (Logan, 1988). If these routes occur in parallel, the current trial's performance will be dependent on whichever process finishes first: when TD is high, episodic retrieval will occur faster than the algorithmic route (Leading to $R T_{\text {Retrieved }}$ ); when TD is low, episodic retrieval will fail with greater likelihood, leaving performance to be achieved by the slower algorithmic route (leading to $R T_{\text {NotRetrieved }}$ ).

The current model does not differentiate between these two accounts. The model bases its predictions on whether the previous task's episodic trace is successfully retrieved or not; it does not specify whether this retrieval is merely "boosting" repetition RT (cf., Horoufchin et al., 2011a) or whether it is beating a slower retrieval process occurring in parallel (cf., Logan, 1988).

To achieve the formal instantiation of Horoufchin et al. (2011a)'s hypothesis, Brown et al. (2007)'s SIMPLE model of memory was used to calculate retrieval probability of episodic traces based on their temporal distinctiveness (as influenced by the RCI ratio). SIMPLE has successfully been applied to a number of serial-order and free-recall memory studies (see for example Brown et al., 2007; Neath \& Brown, 2012); however, to my knowledge this is the first instantiation of SIMPLE to explain RCI effects in task switching. 


\section{Overview of SIMPLE}

In this section, a brief overview of SIMPLE in a task switching context is presented. The discussion refers to two tasks presented in the recent past ( $n-2$ and $n-1)$; the cue for the current trial (n) signals a task repetition, and thus a retrieval attempt is made of the episodic trace at $\mathrm{n}-1$. Based on the RCI ratio, the trace will have a certain "distinctiveness" in episodic memory - i.e. a measure of how isolated the trace is in psychological space from the interfering task at $\mathrm{n}-2$; this distinctiveness directly affects the retrieval probability of that trace. The model assumes that traces is episodic memory are log-compressed: given consistent presentation rates between individual traces, items presented further back in time will be more clustered in psychological space than more recent items.

Distinctiveness is closely related to a trace's similarity - also called confusibility by Brown et al. (2007) - to other traces in psychological space. In the full SIMPLE model, traces can differ along more than one dimension; however, when only temporal dimensions are involved - as is the case in the current study - similarity of the instances at n-1 (item i) and $n-2$ (item $\mathrm{j}$ ) can be calculated by

$$
\eta_{i, j}=\left(\frac{T_{i}}{T_{j}}\right)^{c}
$$

where $T_{i}$ is the temporal age of the more recent trace and $T_{j}$ is the temporal age of the less recent item. Temporal age is calculated as viewed from the current time. For example, if there was an RCI of $100 \mathrm{~ms}$ between $\mathrm{n}-2$ and $\mathrm{n}-1$ and $1,000 \mathrm{~ms}$ between $\mathrm{n}-1$ and $\mathrm{n}, T_{i}$ is $1,000 \mathrm{~ms}$ and $T_{j}$ is $1,100 \mathrm{~ms}$. In Equation $1, c$ is a free parameter which scales the similarity values.

Based on the similarity values, an item's discriminability $\left(D_{i}\right)$ can be calculated. In the current instantiation of SIMPLE, $D_{i}$ basically refers to how isolated the target trace at $\mathrm{n}-1$ is in psychological space in relation to the interfering trace at $\mathrm{n}-2$; higher values reflect greater isolation, and hence greater discriminability. $D_{i}$ is given by

$$
D_{i}=\frac{1}{\sum_{j=1}^{n} \eta_{i, j}}
$$

where $n$ indexes over all items in the memory array (in this case, the task instances at $n-1$ and $\mathrm{n}-2)$. As in item's similarity to itself - that is, $\eta_{i, i}$-is 1 , Equation 2 becomes

$$
D_{i}=\frac{1}{\eta_{i, j}+1}
$$

Given an item's discriminability, its probability of successful retrieval, $p\left(R_{i}\right)$, at time of recall (i.e. when the cue for the current trial is presented) is given by a sigmoid function

$$
p\left(R_{i} \mid D_{i}\right)=\frac{1}{1+e^{-s\left(D_{i}-t\right)}}
$$

where $s$ and $t$ are free parameters which describe the slope of the transforming function and threshold of retrieval, respectively (see Brown et al., 2007, for further justification of this function). Equation 4 shows that higher levels of distinctiveness (or discriminability, 
$D_{i}$ ) lead to higher probabilities of recall, and that this recall probability reaches asymptote at high levels of $D_{i}$.

\section{Model Implementation \& Results}

The model presented here only focussed on task repetition RTs, as the verbal theory of Horoufchin et al. (2011a) only applies to task repetition trials. The modelling aimed to reproduce the decrease in repetition $\mathrm{RT}$ with increasing RCI ratio; model fitting was performed on an individual-participant basis using the data from Horoufchin et al. (2011a, Experiment 1, Group 1). For each RCI ratio, the temporal distances - i.e. $T_{i}$ and $T_{j}$-were calculated $^{4}$. Based on this, the similarity between the two items can be calculated (Equation 1), which then allows calculation of the distinctiveness of the target trace (Equation 3) and its retrieval probability (Equation 4). The response time for the current trial was modelled either as a successful retrieval attempt ( $\left.R T_{\text {Retrieved }}\right)$ or an unsuccessful retrieval attempt $\left(R T_{\text {NotRetrieved }}\right)$. Instead of having $R T_{\text {Retrieved }}$ and $R T_{\text {NotRetrieved }}$ as free parameters, the values were constrained by each participant's data in the group. Specifically, $R T_{\text {Retrieved }}$ was constrained to match each participant's average response time for the two highest RCI ratio conditions (assumed by Horoufchin et al., 2011b, to reflect asymptote TD retrieval) which were 10 and $20 ; R T_{\text {NotRetrieved }}$ was constrained to match each participant's average response time for the two lowest RCI ratio conditions (reflecting complete lack or retrieval) which were 0.05 and 0.1 . The average of the two lowest/highest ratios was used instead of the absolute lowest/highest to try and alleviate some of the noise associated with these responses.

Thus, based on the recall probability of the target episodic trace and the fixed RT parameters, the model's prediction for each RCI ratio is given by

$$
R T=R T_{\text {Retrieved }}+\left(\left[R T_{\text {NotRetrieved }}-R T_{\text {Retrieved }}\right] * 1-p\left(R_{i} \mid D_{i}\right)\right)
$$

The model was implemented in Excel using the solver routine to reduce the RMSD between model predictions and observed data for the RCI ratio repetition RTs. The fitting procedure constrained $t$ to be below 0.99 , and $s$ to be below 30. Although there is no known limit for $s$, not constraining this parameter led to very large estimates for it for some participants. The improvement of fit leaving $s$ unconstrained was negligible, so I retained the constraint to keep it within the range of previously published values of $s$ (see for example Brown et al., 2007). The best fitting model parameters were: $c=4.03[2.06,6.00], s=$ $17.01[11.02,23.00]$, and $t=0.73[0.60,0.86]$; the fixed parameters were $R T_{\text {Retrieved }}=760.67$ $[687.34,834.66]$ and $R T_{\text {NotRetrieved }}=947.42[859.95,1035.39]$. The model fit to the data was satisfactory, with RMSD $=42.97$ and $R^{2}=.94$.

\footnotetext{
${ }^{4}$ It should be noted that only one set of temporal "ages" were calculated for each RCI ratio, even though multiple ages are evident for some RCI ratios. For example, an RCI ratio of 1 arises from sequential RCIs of 100-100, 200-200, 1,000-1,000, or 2,000-2,000. Thus, the value for $T_{i}$ in this example ranges from $100 \mathrm{~ms}$ to $2,000 \mathrm{~ms}$. But, all that matters is the ratio of the RCIs; for example, the verbal theory (and the SIMPLE instantiation) predicts the same retrieval probability for all of the different conditions that give rise to an RCI ratio of 1 . Thus, to avoid redundancy, the model only used one exemplar of temporal age for each RCI ratio, without loss of generality.
} 
Table A1

Examples of retrieval probabilities for each RCI when the RCI ratio is at unity in SIMPLE. See text for details. $c, s$, and t values used are arbitrary.

\begin{tabular}{ccccccc}
\hline RCI n-1 (i) & RCI n-2 (j) & $T_{i}$ & $T_{j}$ & $\eta_{i, j}$ & $D_{i}$ & $p\left(R_{i} \mid D_{i}\right)$ \\
\hline 100 & 100 & 100 & 200 & 0.07 & 0.94 & 0.98 \\
200 & 200 & 200 & 400 & 0.07 & 0.94 & 0.98 \\
1000 & 1000 & 1000 & 2000 & 0.07 & 0.94 & 0.98 \\
2000 & 2000 & 2000 & 4000 & 0.07 & 0.94 & 0.98 \\
\hline
\end{tabular}

\section{Why Temporal Distinctiveness Cannot Account For Slowing with RCI at Ratio- Unity}

As mentioned in the main body of the paper, a temporal distinctiveness account cannot account for the observed slowing of repetition RT with increasing RCI when the RCI ratio is at unity (1). This is because when the RCIs at $n-2$ and $n-1$ match, regardless of the absolute values the resulting similarity scores (Equation 1) will be identical. As a result, the Distinctiveness of a trace (Equation 3) and the retrieval probability (Equation 4) will be identical, leading to identical RT. See Table A1 for a worked example using the absolute RCI values from Group 1.

Appendix B

Modelling Temporal Distinctiveness with Time-Based Decay

The model used the sub-symbolic equations that govern declarative memory retrieval dynamics in the ACT-R cognitive architecture (Anderson, 2007). Declarative memory in ACT-R consists of "chunks" of discrete knowledge. Although successful performance of a task in an experimental context will consist of retrieval of several chunks (Altmann \& Gray, 2008; Grange, Juvina, \& Houghton, 2013) - for example, one chunk might code the relationship between a cue percept and its meaning in the experimental context (e.g. that a dollar sign cues a form judgement), and another might code the relevant response rules (e.g. "letter" is paired with a left key press) - the present work only modelled the retrieval dynamics of a task being represented by a single chunk.

In ACT-R, a retrieval request to memory returns the most active among all chunks stored. At retrieval, transient noise is present in the system that makes chunk retrieval stochastic. Each chunk's activation in memory is related to its "match" to the current context, as well as a "base" level of activation (which can be thought of as its resting levels of activation) which reflects recency and frequency of practice a chunk experiences. It is this base-level activation that I use to model the dynamics of task performance in the current experiment. A chunk's activation $\left(A_{i}\right)$ level is given by

$$
A_{i}=\ln \left(\sum_{j=1}^{n} t_{j}^{-d}\right)
$$

where the summation is across all presentations (n) of a chunk, $t_{j}$ is the time since the $j^{\text {th }}$ presentation of that chunk, and $d$ is an architectural decay parameter fixed at 0.5 . This equation demonstrates that as a chunk becomes more practiced, its activation level 
increases; importantly to the current modelling, this activation decays as a function of time since the chunk was presented.

As this equation is computationally expensive (and requires simulation), an approximation for a chunk's activation as a function of time $(t)$ since its last presentation is given by

$$
A_{i}=-0.5 * \ln (t) .
$$

A chunk's activation level directly affects the speed with which it can be retrieved: the more active a chunk, the faster it can be retrieved and acted upon. The relation between response time (RT) and activation is given by

$$
R T=F * \exp \left(-A_{i} / s\right)
$$

where $F$ is a latency scaling parameter, and $s$ is activation noise; $F$ and $s$ are the only free parameters in the model. Equations 7 and 8 state that as the time since a task was last performed increases (as a function of RCI), that task's representation in memory will become less active, requiring more time to retrieve and perfom that task when it becomes relevant again. This model clearly predicts slower RTs at longer RCIs for task repetitions, as the relevant task will decay as a function of time. Thus, this structure is well suited to test whether a purely time-based process can reproduce the pattern of data associated with temporal distinctiveness.

\section{Modelling Implementation}

The model was implemented in Excel using the solver routine to minimise the rootmean-square deviation (RMSD) between model predictions and observed data for task repetition RTs as a function of RCI ratio. The model fitting was performed for Group 1's data from Experiment 1 of Horoufchin et al. (2011a) data; all model fitting was performed on individual participant data. The model predictions were aggregated across individuals for each group and data set for presentation purposes.

The model calculated estimated activation levels for task chunks for participants as a function of a current trial's absolute RCI for each group using Equation 7; thus, model predictions for each RCI were first calculated. RTs were estimated using Equation 8, and then these predictions were extrapolated onto RCI ratios based on the current trial RCIs that make up each RCI ratio (See Table 1). For example, in Group 1, an RCI ratio of 0.1 can occur from two possible RCIs on the current trial (i.e. $1,000 \mathrm{~ms}$ and $2,000 \mathrm{~ms}$ ); final model predictions for an RCI ratio of 0.1 for a participant in this group was therefore the average of the model's predictions for RCIs of $1,000 \mathrm{~ms}$ and $2,000 \mathrm{~ms}$.

The model fitting procedure estimated the two free parameters $F$ and $s$ for each participant by minimising the RMSD between model predictions and observed data for the RCI ratio RTs. The best fitting parameters were $F=524.50[454.54,594.46]$ and $s=7.22$ [5.21, 9.22].

The model fit was $\mathrm{RMSD}=49.00$ and $R^{2}=.87$, which is worse than the temporal distinctiveness model, but the decay model had only two free parameters (compared to three for the TD model). 
Appendix C

Accounting for Prior RCIs with Temporal Distinctiveness

When accounting for three prior RCIs, the temporal distinctivenness of a target memory trace $\left(D_{i}\right)$ is related to its confusibility in psychological space to itself $\left(\eta_{i i}\right)$, its confusibility to the task at $\mathrm{n}-1\left(\eta_{i j}\right)$ and its confusibility to the task at $\mathrm{n}-2\left(\eta_{i k}\right)$. With four RCIs $(100 \mathrm{~ms}$, $200 \mathrm{~ms}, 1000 \mathrm{~ms}$, and $2000 \mathrm{~ms}$ ), Table C1 shows the temporal distinctiveness of a target trace $\left(D_{i}\right)$ when the RCI-raio is at unity (that is, the current RCI and the previous RCI match) for all four values of current-RCI.

The calculation of $D_{i}$ will be elaborated for the first row of Table C1 to show how the calculations are made. In this first example, the temporal age of $i$ is 2000ms, the temporal age of $j$ is $4000 \mathrm{~ms}$, and the temporal age of $k$ is $4100 \mathrm{~ms}$. Thus, using Equation 1 in Appendix A, we can calculate the confusibility between $i$ and every other task in memory (with a limit of 3 prior tasks: $i, j$, and $k$ ):

$$
\begin{gathered}
\eta_{i, i}=\left(\frac{T_{i}}{T_{i}}\right)^{c}=\left(\frac{2000}{2000}\right)^{4.03}=1 \\
\eta_{i, j}=\left(\frac{T_{i}}{T_{j}}\right)^{c}=\left(\frac{2000}{4000}\right)^{4.03}=0.06 \\
\eta_{i, k}=\left(\frac{T_{i}}{T_{k}}\right)^{c}=\left(\frac{2000}{4100}\right)^{4.03}=0.06
\end{gathered}
$$

where $c=4.03$, taken from the best fitting parameters reported in Appendix A.The distinctiveness of $i, D_{i}$ is the reciprocal of the summed confusibility values (Equation 2):

$$
D_{i}=\frac{1}{\sum_{j=1}^{n} \eta_{i, j}}=\frac{1}{1+0.06+0.06} \approx 0.89
$$

(note the final $D_{i}$ is slightly different than in the table due to rounding). All that changes with increasing current-RCI is the confusibility between the target trace $i$ and the trace from n-3k, expressed in the $\eta_{i, k}$ values. The effect of $\mathrm{n}-3$ has greater average effect on $D_{i}$ when the current RCI is larger. 
Table C1

Demonstration of how distinctiveness of the previous trial's trace increases when the $R C I$ from $n-3$ increases, even when the RCI-ratio between $n-2$ and $n-1$ is at unity.

\begin{tabular}{ccccccc}
\hline RCI n-3 (k) & RCI n-2 (j) & RCI n-1 (i) & $\eta_{i i}$ & $\eta_{i j}$ & $\eta_{i, k}$ & $D_{i}$ \\
\hline 100 & 2000 & 2000 & 1 & 0.06 & 0.06 & 0.896 \\
200 & 2000 & 2000 & 1 & 0.06 & 0.05 & 0.9 \\
1000 & 2000 & 2000 & 1 & 0.06 & 0.02 & 0.921 \\
2000 & 2000 & 2000 & 1 & 0.06 & 0.01 & 0.932 \\
& & & & & & \\
100 & 1000 & 1000 & 1 & 0.06 & 0.05 & 0.9 \\
200 & 1000 & 1000 & 1 & 0.06 & 0.04 & 0.907 \\
1000 & 1000 & 1000 & 1 & 0.06 & 0.01 & 0.932 \\
2000 & 1000 & 1000 & 1 & 0.06 & 0 & 0.939 \\
& & & & & & \\
100 & 200 & 200 & 1 & 0.06 & 0.02 & 0.921 \\
200 & 200 & 200 & 1 & 0.06 & 0.01 & 0.932 \\
1000 & 200 & 200 & 1 & 0.06 & 0 & 0.942 \\
2000 & 200 & 200 & 1 & 0.06 & 0 & 0.942 \\
& & & & & & \\
100 & 100 & 100 & 1 & 0.06 & 0.01 & 0.932 \\
200 & 100 & 100 & 1 & 0.06 & 0 & 0.939 \\
1000 & 100 & 100 & 1 & 0.06 & 0 & 0.942 \\
2000 & 100 & 100 & 1 & 0.06 & 0 & 0.942 \\
\hline
\end{tabular}

